Chem. Ber. 118, 979-999 (1985)

\title{
Substituierte Benzobenzvalene und Diazabenzobenzvalene - Synthesen aus Diels-Alder-Addukten des Benzvalens und NMR-Spektroskopie
}

\author{
Manfred Christl* und Silke Freund ${ }^{1)}$ \\ Institut für Organische Chemie der Universität Würzburg, \\ Am Hubland, D-8700 Würzburg
}

Eingegangen am 29. Februar 1984

\begin{abstract}
Die Diels-Alder-Additionen von Benzvalen (4) an 3,5-Dichlor-, 3,4,5-Trichlor- und Tetrachlor- $\alpha$ pyron liefern die $\delta$-Lactone 8 - 10, welche beim Erhitzen Kohlendioxid abspalten und in die chlorsubstituierten 1,3-Cyclohexadien-Derivate 11- 13 ubergehen. Beim Belichten schnuren 7, 12 und 13 einen Cyclobutenring ab, wobei die zwei stereoisomeren Pentacyclo[4.4.0.0.0.4. $\left.0^{3.5} \cdot 0^{7,10}\right] \mathrm{dec}-8$ ene 14a, b bzw. ihre Chlorderivate 15a, b und 16a, b entstehen. 14a, b sind zwei neue Mitglieder $\operatorname{der}(\mathrm{CH})_{10}$-Familie. Erhitzen von $14 \mathrm{a}, \mathrm{b}$ und $16 \mathrm{a}, \mathrm{b}$ auf $120^{\circ} \mathrm{C}$ macht die photochemische Isomerisierung rückgängig. Als 1,3-Butadien-Abkömmling ist 13 zur [4 + 2]-Cycloaddition mit Cyclopropen und 4-Methyl-4H-1,2,4-triazol-3,5-dion befähigt. - Mit Kalium-tert-butoxid in Dimethylsulfoxid gelingt unter Chlorwasserstoffeliminierung die Aromatisierung von 11-13 zu den chlorsubstituierten Benzobenzvalenen 19-22. Die Behandlung von 10 mit Natriummethoxid führt zur Bildung des Benzobenzvalencarbonsäure-methylesters 27. Verschiedene Oxidationsmittel wandeln die Dihydropyridazine 28 und 30 in die Diazabenzobenzvalene 29 bzw. 31 um. Bei 28 läuft diese Dehydrierung, wahrscheinlich durch eine Säurekatalyse ausgelöst, schon mit Luftsauerstoff ab, der jedoch in erheblichem Ausmaß den Übergang in cis-3,4-Dibenzoyltricyclo[3.1.0.0 2,6]hexan (32) bewirkt. - Die chemischen Verschiebungen der Bicyclo[1.1.0]butanBrückenkopf-CH-Gruppen des Benzobenzvalens (6) und seiner Derivate, die im Vergleich zu gewöhnlichen Bicyclo[1.1.0]butan-Abkommlingen sehr große Werte aufweisen, werden anhand eines Orbitalwechselwirkungsmodells gedeutet.
\end{abstract}

Substituted Benzobenzvalenes and Diazabenzobenzvalenes - Syntheses from Diels-Alder Adducts of Benzvalene and NMR Spectroscopy

The Diels-Alder additions of benzvalene (4) to 3,5-dichloro-, 3,4,5-trichloro-, and tetrachloro- $\alpha$ pyrone give the $\delta$-lactones $8-10$, which eliminate carbon dioxide on heating to form the chlorosubstituted 1,3-cyclohexadiene derivatives $11-13$. On irradiation, 7, 12, and 13 undergo a cyclobutene ring closure leading to the two stereoisomeric pentacyclo[4.4.0.0 $\left.0^{2,4} \cdot 0^{3,5} \cdot 0^{7,10}\right] \mathrm{dec}-8$-enes $14 \mathrm{a}, \mathrm{b}$ and their chloro derivatives $15 \mathrm{a}, \mathrm{b}$ and $16 \mathrm{a}, \mathrm{b}$, respectively. The compounds $14 \mathrm{a}, \mathrm{b}$ are new members of the $(\mathrm{CH})_{10}$ family. Heating of $14 \mathrm{a}, \mathrm{b}$ and $16 \mathrm{a}, \mathrm{b}$ to $120^{\circ} \mathrm{C}$ reverses the photochemical isomerization. As a 1,3-butadiene derivative, 13 takes up cyclopropene and 4-methyl-4H-1,2,4triazole-3,5-dione in [4+2] cycloadditions. - Potassium tert-butoxide in dimethyl sulfoxide brings about the aromatizations of $11-13$ with elimination of hydrogen chloride and formation of the chloro-substituted benzobenvalenes $19-22$. The treatment of 10 with sodium methoxide leads to methyl benzobenzvalenecarboxylate 27 . Several oxidizing reagents transform the dihydropyridazines 28 and 30 into the diazabenzobenzvalenes 29 and 31, respectively. Probably promoted by an acid catalysis this dehydrogenation of 28 is accomplished even by air, which to a considerable extent gives rise also to cis-3,4-dibenzoyltricyclo[3.1.0.0 $\left.0^{2,6}\right]$ hexane (32), however. -

(C) VCH Verlagsgesellschaft mbH, D-6940 Weinheim, 1985

$0009-2940 / 85 / 0303-0979 \$ 02.50 / 0$ 
Compared to those of usual bicyclo[1.1.0]butane derivatives the bridgehead $\mathrm{CH}$ groups of benzobenzvalene (6) and its derivatives reveal remarkable low field NMR chemical shifts, which are interpreted in terms of an orbital interaction model.

Das ${ }^{13} \mathrm{C}$-NMR-Spektrum des Benzvalens (4) fuhrte uns zur Entdeckung eines nach tiefem Feld gerichteten Effekts, der generell an der C-4-Resonanz von Cyclopentenen mit festgehaltener Briefumschlagkonformation beobachtbar ist ${ }^{2}$. Es handelt sich dabei um ein von Norbornen und Norbornadien her bekanntes Phänomen. Dort absorbiert $C-7$ bei $\delta=48.5$ bzw. 75.4, wăhrend der entsprechende Wert des Norbornans (1) mit 38.7 bei vergleichsweise hohem Feld liegt.

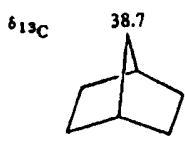

1<smiles>OC1CCC2CC1c1ccccc12</smiles>

2<smiles>[CH]1C2C=CC1c1ccccc12</smiles>

3

Wie die Daten ${ }^{3)}$ von Benzonorbornen (2) und Benzonorbornadien (3) zeigen, tritt dieser Tieffeldeffekt auch bei konformativ fixierten Benzocyclopenten-Derivaten auf. Dagegen ist beim quasi-ebenen Indan die betreffende chemische Verschiebung $(\delta=25.3)$ gegenuber dem Cyclopentan-Wert $(\delta=26.3)$ sogar um $1 \mathrm{ppm}$ nach hohem Feld verändert.

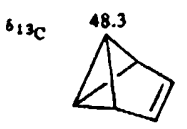

4

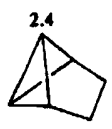

5

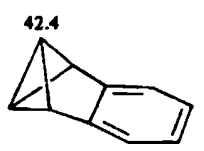

6<smiles></smiles>

7

Bei der Korrektur unserer Arbeit über $4^{2}$ erwähnten wir den Tieffeldeinfluß des aromatischen Teils von Benzobenzvalen (6) auf die Absorption der Bicyclo[1.1.0]butan-Brückenkopf-C-Atome. Relativ zur gesättigten Vergleichsverbindung Tricyclo[3.1.0.0.6.6 40.0 ppm eine Differenz, die dem 45.9-ppm-Effekt im Benzvalen-Spektrum nahe kommt. Daß die Anellierung von vier $C$-Atomen an das Gerüst von 5 allein mit der Tieffeldverschiebung der betreffenden 6-Resonanz nichts zu tun hat, beweisen die entsprechenden Daten des Dihydroderivats $7^{4 a)}$ und des Hexahydroderivats) von 6 . Um das bei 6 zutage tretende Phänomen auf eine breitere Basis zu stellen und es auch im Licht der Derivatisierung von $6 \mathrm{zu}$ sehen, synthetisierten wir die Titelverbindungen.

\section{A. Neue Diels-Alder-Addukte des Benzvalens (4)}

Unseren früheren $[4+2]$-Additionen ${ }^{4)}$ mit 4 stellen wir jetzt jene von chlorsubstituierten $\alpha$-Pyronen an die Seite. Die Dien-Aktivitat des unsubstituierten $\alpha$-Pyrons ist seit den Arbeiten von Diels und Alder6) bekannt, jedoch nicht hoch, wie die Anlagerung von Maleinsäureanhydrid erst bei $115^{\circ} \mathrm{C}$ beweist. Wir fanden, daß mit 4 keine Reaktion eintritt. Offensichtlich kompensieren im $\alpha$-Pyron Donor- und Akzeptorsubstituent in Übereinstimmung mit dem Grenzorbitalmodell?) ihre an sich aktivierende Wirkung. Daruber hinaus dürte die Konjugation zwischen diesen Substituenten zu einer Grundzustandsstabilisierung (Pyrylium-2-olat-Grenzformeln) und so zu einer Minderung der Dienaktivităt führen. Märkl ${ }^{8)}$ beobachtete ein Absinken der Reaktivităt gegenüber Maleinsäureanhydrid, wenn der $\alpha$-Pyronring eine zunehmende Zahl von Chlorsubstituenten trăgt. Zwei Patente ${ }^{9)}$ beschreiben neben der Addition von Maleinsäureanhydrid an Tetrachlor- $\alpha$-pyron auch jene von Methylacrylat, Cyclopentadien, Dicyclopentadien und Norbornen sowie Anwendungen derartiger Cycloadditionen in der Polymerenchemie. Schuster und Sauer 10)

Chem. Ber. 118 (1985) 
erhielten bei der Umsetzung von Tetrachlor- $\alpha$-pyron mit 1,1'-Dimethylbi-2-cyclopropen-1-yl ein stabiles Primăraddukt und ein Snouten-Derivat, das durch rasche Eliminierung von Kohlendioxid aus einem zweiten Primäraddukt und anschließende intramolekulare Diels-Alder-Addition entsteht.

$\mathrm{Daß}$, wie schon von $\mathrm{Markl}^{8)}$ vermutet, die Chlorsubstituenten die Diels-Alder-Additionen zu solchen mit inversem Elektronenbedarf machen, beweist die glatte Anlagerung von 3,5-Dichlor-, 3,4,5-Trichlor- und Tetrachlor- $\alpha$-pyron an 4 unter Bildung der Addukte 8, $9 \mathrm{bzw} .10^{11 \mathrm{a}}$. Wie erwartet reagierte 3,5-Dichlor- $\alpha$-pyron am langsamsten $\left(24 \mathrm{~h}\right.$ bei $20^{\circ} \mathrm{C}$ ), während zwischen 3,4,5-Trichlor- $(4 \mathrm{~h})$ und Tetrachlor- $\alpha$-pyron $(3 \mathrm{~h})$ kaum ein Unterschied beobachtet wurde.

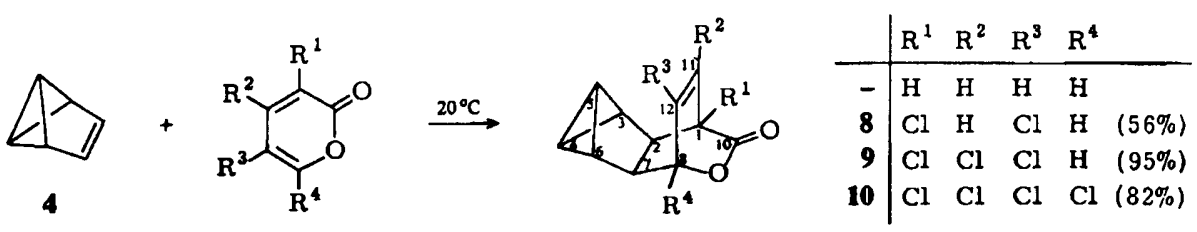

Die Konstitution von 8 - 10 ergibt sich zweifelsfrei aus den analytischen und spektroskopischen Daten. Im ${ }^{1}$ H-NMR-Spektrum von 8 und 9 absorbiert jeweils 7-H bei tieferem Feld als 2-H, was eindeutig aus $J_{7,8}=4.2 \mathrm{~Hz}$ folgt. Wahrscheinlich wird der Hochfeldwert von 2-H durch den Anisotropieeffekt der Carbonylgruppe verursacht, womit die analoge Zuordnung der Signale von 2-H und 7-H auch im Spektrum von 10 naheliegt. Bezüglich der Bandencharakterisierung beim Tricyclo[3.1.0.02,6]hexan-System verweisen wir auf frühere Arbeiten $\left.{ }^{4 a}, 11 b\right)$. Die Konfiguration von 8 folgt aus der Fernkopplung $J_{2,11}=1.1 \mathrm{~Hz}$, womit die antiperiplanare Anordnung von 2-H und C-11 angezeigt ist. Legt man die Analogie zu den Kopplungsverhăltnissen in Norbornen zugrunde, so scheidet eine Vertauschung von Chlorvinylen- und Lactonbrücke aus, und dies ist auch für 9 und 10 wahrscheinlich. Die angegebene Stereochemie geht parallel zu jener der 0 -Chinon-Addukte an 4 4a), wo der sich auf ${ }^{1} H$-NMR-Daten der zugehörigen Chinoxaline gründende Vorschlag durch Röntgenstrukturanalysen Bestătigung fand ${ }^{12}$.

Wie von anderen $\alpha$-Pyron-Diels-Alder-Addukten bekannt ist ${ }^{6,8.9,11 a)}$, eliminierten die Lactone 8-10 leicht Kohlendioxid. In einer Deuterochloroformlösung von 10 stellten wir bei $20^{\circ} \mathrm{C}$ eine Halbwertszeit von ca. einer Woche für die Bildung von 13 fest. Zur präparativen Darstellung der 1,3-Cyclohexadien-Derivate 11-13 erhitzten wir 8-10 auf etwa $150^{\circ} \mathrm{C}$.

$$
\begin{aligned}
& \text { 8-10 } \underset{-\mathrm{CO}_{2}}{\stackrel{\Delta T}{\longrightarrow}} \\
& \qquad \begin{array}{l|lllll}
\mathrm{R}^{1} & \mathrm{R}^{2} & \mathrm{R}^{3} & \mathrm{R}^{4} \\
\hline 12 & \mathrm{Cl} & \mathrm{H} & \mathrm{Cl} & \mathrm{H} \\
\mathbf{C l} & \mathrm{Cl} & \mathrm{Cl} & \mathrm{H} \\
\mathrm{Cl} & \mathrm{Cl} & \mathrm{Cl} & \mathrm{Cl}
\end{array}
\end{aligned}
$$

Chem. Ber. 118 (1985) 
Verbindung 13 war erstmals durch Photolyse des Tetrachlor-o-chinon-Addukts an 4 dargestellt worden ${ }^{4}$. Einen dritten Weg zu 13 fanden wir in der Umsetzung von 4 mit Tetrachlorthiophen-1,1-dioxid, wobei die nicht beobachtete Zwischenstufe sofort Schwefeldioxid eliminierte. Raasch ${ }^{13)}$ hatte diesen Heterocyclus als vielseitiges Dien in Diels-Alder-Additionen mit inversem Elektronenbedarf genutzt.

\section{B. Photolyse und Diels-Alder-Reaktionen der Tetracyclo[4.4.0.0 $\left.2,4.0^{3,5}\right]$ deca- 7,9-diene}

Sowohl 7 als auch 11-13 verlockten zum Versuch, durch Abspaltung von Benzol bzw. Di-, Tri- und Tetrachlorbenzol das Bicyclo[1.1.0]butan-2,4-diyl-Diradikal zu erzeugen, das eine mögliche Vorstufe für Tetrahedran ist. Bei der Photolyse von 7, 12 und 13 mußten wir aber den trivialen Ringschlu $\beta^{14)}$ der 1,3-Diensysteme zu den cisanellierten Cyclobuten-Derivaten 14-16 zur Kenntnis nehmen ${ }^{15)}$. Das exo-endo-Isomerenverhăltnis, in dem die Pentacyclen 14-16 anfielen, scheint vom Raumanspruch von $R^{1}$ und $R^{4}$, den Substituenten der Brückenköpfe 7 und 10, abzuhängen. Zwei kleine Atome $\left(R^{1}=R^{4}=H\right)$ hatten $14 a: 14 b=84: 16$, ein großes und ein kleines $\left(R^{1}=\mathrm{Cl}, \mathrm{R}^{4}=\mathrm{H}\right)$ 15a: 15b $=63: 37$ und zwei große $\left(\mathrm{R}^{1}=\mathrm{R}^{4}=\mathrm{Cl}\right) 16 \mathrm{a}: 16 \mathbf{b}=$ 40:60 zur Folge. Erhitzen auf $120^{\circ} \mathrm{C}$ führte bei 14 und 16 zur Rückbildung von 7 bzw. 13. Die unsubstituierten Verbindungen $14 \mathbf{a}, \mathbf{b}$ sind neue Valenzisomere der $(\mathrm{CH})_{10^{-}}$ Familie.

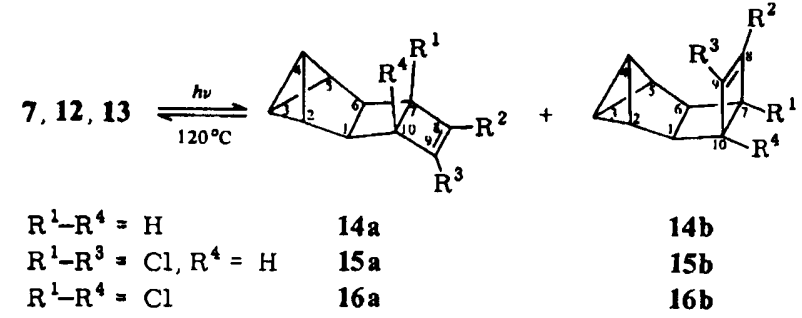

Durch die Kopplung $J_{1,10}$ gelang die Unterscheidung der Stereoisomeren 15a und 15b. Der Verbindung mit $6.7 \mathrm{~Hz}$, einem Wert im typischen Bereich der Kopplungskonstanten cis-vicinaler Protonen, muß die Konfiguration $15 \mathrm{~b}$ zugeordnet werden, während $J_{1,10}$ beim anderen Isomeren (15a) nicht aufgelöst ist. Im ${ }^{13} \mathrm{C}$-NMR-Spektrum erscheinen die meisten Absorptionen des sterisch gedrängteren $15 \mathrm{~b}$ im Vergleich zu jenen von $15 \mathbf{a}$ bei hoherem Feld. Auf dieser Basis wurden auch bei 14a, b und 16a, b Zuordnung und Isomerenverhăltnis bestimmt.

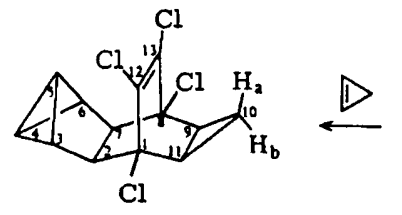

17

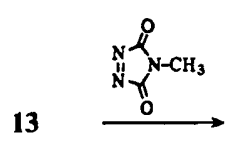

13

18

Einige Versuche wurden mit $\mathbf{1 3}$ im Hinblick auf seine Dienaktivität in Diels-AlderReaktionen unternommen. Die Benzvalen-Doppelbindung war nicht attraktiv genug, 
jedoch fielen mit Cyclopropen und 4-Methyl-4H-1,2,4-triazol-3,5-dion die 1:1-Addukte 17 bzw. 18 mit guten Ausbeuten an. In 17 ist die Orientierung des Cyclopropanrings (Stereochemie an C-9, -11) nicht belegt, aber aus sterischen Gründen wahrscheinlich.

\section{Darstellung der Benzobenzvalen-Derivate}

Das Syntheseprinzip für Benzvalen (4) wurde von Katz et al., seinen Entdeckern, auch auf Benzobenzvalen (6) übertragen, das damit aus dem Indenyl-Anion und Chlorcarben erhältlich ist ${ }^{16)}$. Burger et al. erarbeiteten weitere Anwendungen wie die Darstellung der AnthracenValenzisomeren Naphtho[2,3]benzvalen und sym-Benzvaleno-benzobenzvalen aus dem Dianion des s-Indacens ${ }^{17)}$ und des Pyrrolo-3-azabenzvalens aus dem 4-Azapentalen-Anion ${ }^{18)}$. Der Einsatz von Dilithiopentalen erbrachte neben anderen tetracyclischen Benzvalenabkömmlingen das schon bekannte 6, aber keines der möglichen Naphthalin-Valenzisomeren mit zwei Bicyclo[1.1.0]butanSystemen ${ }^{19)}$.

Eine zweite bisher erforschte Möglichkeit, das Benzobenzvalen-Gerüst aufzubauen, lehnt sich an den photochemischen Weg zum Benzvalen und einigen Derivaten an ${ }^{4 b)}$. So stellten Yoshida et al. 20a) am Bicyclo[1.1.0]butan-System dreifach tert-butylsubstituierte Benzobenzvalene durch Belichten entsprechender Naphthaline her und gingen der Frage nach, ob dieser Reaktionstyp, gekoppelt mit der hochexothermen Ruckisomerisierung, zur Nutzung von Sonnenenergie geeignet sei $^{20 b)}$.

$$
11-13 \underset{\text { Dimethylsulfoxid }}{\operatorname{KoC(\mathrm {CH}_{2})_{3}}}
$$

Ein Weg zu chlorsubstituierten Benzobenzvalenen liegt mit der Dehydrierung der 1,3-Cyclohexadienderivate 11 - 13 auf der Hand. Diesbezügliche Versuche mit 13 führten jedoch nicht zum Erfolg. Zur Aromatisierung von 1,2,3,4-Tetrachlor-1,3-cyclohexadienen hat sich die Behandlung mit Kaliumhydroxid in Ethanol bewährt, wobei 1,2,4-Trichlorbenzole entstehen ${ }^{13.21)}$. Wie man durch Einsatz deuterierter Reagenzien bewies, wird nach einer basenkatalysierten $\mathrm{H}$-Wanderung Chlorwasserstoff eliminiert ${ }^{13)}$. Zwar versagte dieses Verfahren bei 13, aber die Anwendung einer stärkeren Base half weiter. Kalium-tert-butoxid in Dimethylsulfoxid (DMSO) wandelte 13 mit $54 \%$ Ausbeute in ein 5:1-Gemisch der Trichlorbenzobenzvalene 21 und $22 \mathrm{um}$. Im Vergleich zu den früheren Versuchen ist neu, daß zwei Isomere entstehen mit jenem in geringerer Menge, das die erwartete ${ }^{13,21)}$ Verteilung der Chloratome aufweist. Als Beleg für die Position der Chloratome in 21 durfen die ${ }^{13} \mathrm{C}$-NMR-Daten (Abschnitt D) sowie die 0.7-Hz-Kopplung zwischen dem aromatischen und einem benzylständigen Proton gelten. Aufgrund bekannter Wechselwirkungen von Benzylprotonen ${ }^{22)}$ müßte es sich um eine Kopplung über vier Bindungen handeln, was die Zuordnung festlegt (Tab. 1).

Einblick in den Mechanismus gewannen wir durch Verwendung von [ $D_{6}$ ]DMSO. Nicht unerwartet wurden durch das stark basische Reagenz die relativ aciden Protonen der Bicyclo[1.1.0]butan-Bruckenkopfpositionen abgelöst und durch Deuterium ersetzt. Wie Schema 1 zeigt, wurde aber Deuterium auch in die aromatischen Ringe von 21 und

Chem. Ber. $1 / 8(1985)$ 
22 aufgenommen, womit der fur die Reagenzien Kaliumhydroxid/Ethanol beobachtete Ablauf ${ }^{13)}$ auch hier zutrifft. Das Isomerenverhăltnis 21a:22a (und damit 21:22) beruht auf dem Verhältnis der Deuteronenanlagerung an die periphere (zu 25) bzw. die zentrale Position (zu 24) des Pentadienyl-Anion-Systems in 23. Möglicherweise ist der Energievorteil, den 25 wegen der Konjugation der Doppelbindungen gegenubber 24 genießen sollte, für die überwiegende Bildung von 21 a verantwortlich. Daß in den früheren Fällen nur die zu 22 analogen Verbindungen entstanden ${ }^{13,21)}$, liegt wahrscheinlich am anderen Solvens.

Schema 1. Umwandlung von 13 in 21 a und 22 a mit Hilfe von Kalium-tert-butoxid in [D6]Dimethylsulfoxid

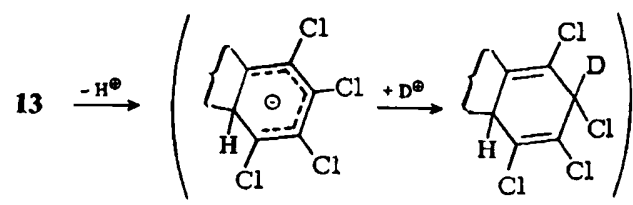

23

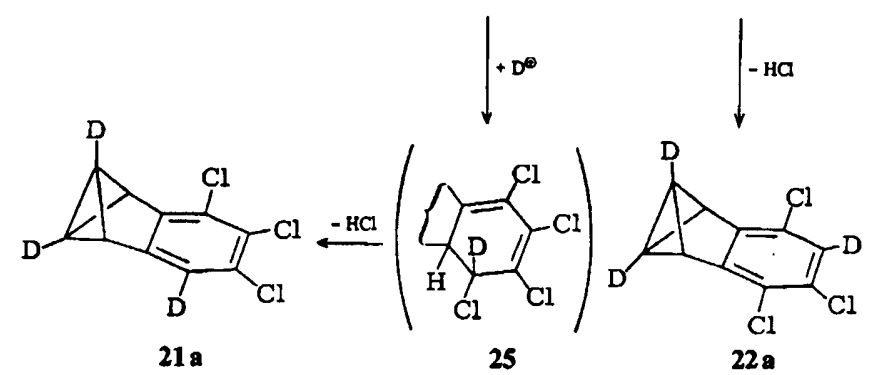

Analog zur Aromatisierung $13 \rightarrow 21+22$ gingen aus 11 und 12 das Monochlorbenzobenzvalen 19 mit $68 \%$ bzw. das Dichlorbenzobenzvalen 20 mit $43 \%$ Ausbeute hervor. Die Stellung der Chloratome folgt wieder zweifelsfrei aus den NMR-Spektren (Abschnitt D). Bei beiden Reaktionen kann jeweils nur die Abtrennung des Protons vom C-1 des Substrats die Reaktion einleiten. Die wegen der Nachbarschaft zu einem Chloratom am C-7 intuitiv als acider zu betrachtende Position 6 dürfte erst bei der $\mathrm{HCl}$-Eliminierung nach erfolgter Isomerisierung ins Spiel kommen.

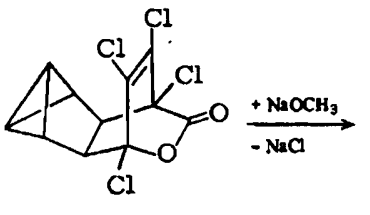

10

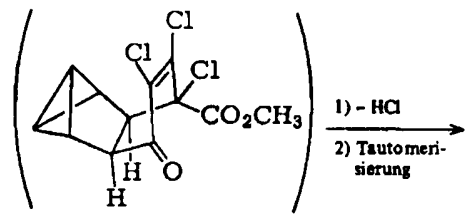

26<smiles></smiles>

27

Eine andere Aromatisierungsreaktion gelang ausgehend vom Lacton 10, aus dem bei der Behandlung mit Natriummethoxid mit 54\% Ausbeute der Dichlorhydroxybenzoesäure-methylester 27 resultierte ${ }^{11 a)}$. Offenbar wandelt das Methoxid-Ion die Lacton- in die Methoxycarbonylfunktion um unter gleichzeitiger Abspaltung eines Chlorid-Ions 
und Generierung der Ketofunktion der Zwischenstufe 26. Chlorwasserstoffeliminierung und Keto-Enol-Tautomerisierung führen dann zum Endprodukt 27.

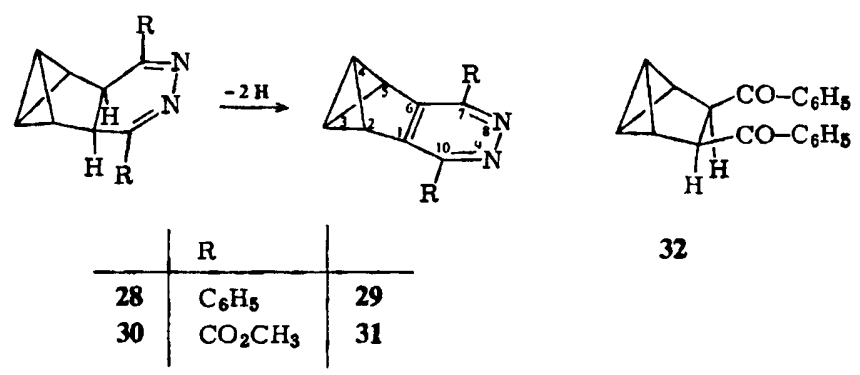

Vor einiger Zeit hatten wir durch Addition von Benzvalen (4) an 3,6-Diphenyl1,2,4,5-tetrazin und 1,2,4,5-Tetrazin-3,6-dicarbonsäure-dimethylester die Dihydropyridazinabkơmmlinge 28 bzw. 30 hergestellt ${ }^{4)}$. Ihre Dehydrierung zu den Diazabenzobenzvalenen 29 bzw. 31 bewerkstelligten mehrere Oxidationsmittel. 5,6-Dichlor-pbenzochinon-2,3-dicarbonitril (DDQ) erbrachte 42 bzw. 60\% Ausbeute. Durch das Reagens Fenchon/Kaliumfencholat ${ }^{23)}$ ging $28 \mathrm{zu} 36 \%$ in 29 uber. Dehydrobenzol bevorzugte an 28 nicht wie erhofft das Diazadiensystem, sondern die 1,6-H-Atome unter Bildung von $44 \%$ 29. Auch Belichten von 28 fuhrte lediglich zur Dehydrierung, und selbst Luftsauerstoff kann diese Reaktion bewirken. In dem Bestreben aus 28 cis-3,4-Dibenzoyltricyclo[3.1.0.0 2,6] hexan (32) zu synthetisieren, folgten wir Literaturbefunden zur Umwandlung von Azinen in die zugrunde liegenden Carbonylverbindungen ${ }^{24 a)}$ und behandelten 28 mit Singulettsauerstoff. Tatsăchlich bildete sich 32; daneben fielen auch wechselnde Mengen 29 an. Kontrollversuche lassen aber bezweifeln, ob Singulettsauerstoff mitwirkt. So erbrachte das Einleiten von Sauerstoff in eine Lösung von 28 in Benzol, Dichlormethan oder Acetonitril ohne Belichten und ohne Sensibilisator ein Gemisch aus 29 und 32 in etwa der gleichen Zeit wie beim Singulettsauerstoffexperiment. Sogar das Rühren von 28-Losungen an der Luft führte zum Erfolg. Die Tatsache, daß in Gegenwart von suspendiertem Kaliumcarbonat 28 nur sehr langsam angegriffen wurde, laßt die Katalyse durch eine während der Reaktion entstehende Săure vermuten. Dehydrierungen von Dihydropyridazinen mit Triplettsauerstoff sind bekannt ${ }^{2 a b}$, jedoch fanden wir fur die Bildung eines Ketons unter diesen Bedingungen kein Vorbild.

Auch zwei Äquivalente Persäure bringen aus Azinen die Carbonylverbindungen hervor ${ }^{25)}$. Dementsprechend erhielten wir aus $28 \mathrm{mit} m$-Chlorperbenzoesäure $30 \% 32$. Die Stereochemie von 32 folgt eindeutig aus der Zahl der Signale in den NMR-Spektren. Wie für die $C_{s}$-Symmetrie erwartet, verursacht das Tricyclo[3.1.0.02,6]hexan-Gerüst vier Signale, während das trans-Isomere aufgrund der $C_{2}$-Symmetrie nur drei ergeben sollte. Die Umwandlung $28 \rightarrow 32$ ließ sich nicht auf das mit stärker elektronenziehenden Substituenten belastete 30 übertragen. Mit Sauerstoff trat keine Reaktion ein, und $m$-Chlorperbenzoesäure dehydrierte $30 \mathrm{zu} 31$.

\section{NMR-Spektren der Benzobenzvalene}

In den ${ }^{1} \mathrm{H}-\mathrm{NMR}$-Spektren der Benzobenzvalene mit $C_{2 v}$-Symmetrie $(6,20,29$ und 31) kơnnen die beiden vom Bicyclo[1.1.0]butan-System stammenden Signale ohne Zusatz- 
experimente (Aufsuchen der ${ }^{13} \mathrm{C}$-Satelliten oder selektive Deuterierung) nicht spezifisch zugeordnet werden, weil die beiden Tripletts der $\mathbf{A}_{2} \mathbf{X}_{2}$-Systeme mit Vicinalkopplungen von $1.5-1.8 \mathrm{~Hz}$ die gleiche Form haben. Die Derivate mit $C_{s}-$ Symmetrie $(19,21,22$ und 27) bieten durch die Unterschiedlichkeit der seitlichen Bicyclo[1.1.0]butan-CHGruppen einen einfachen Ausweg. Tatsächlich zeigen die Spektren von 21, 22 und 27 für diese Protonen zwei Signale mit der typischen Fernkopplung $J_{2,5}=5.4-5.7 \mathrm{~Hz}$. Damit steht fest, und das darf auch für die Verbindungen der höheren Symmetrie angenommen werden, daß 3,4-H bei erheblich tieferem Feld $(\Delta \delta=1.17-1.57 \mathrm{ppm})$ absorbieren als 2-H und 5-H (Tab. 1). Dies befindet sich im Gegensatz zur Reihenfolge der chemischen Verschiebungen in gewöhnlichen Bicyclo[1.1.0]butanen, wie der Vergleich mit Verbindungen aus den Abschnitten $\mathrm{A}$ und $\mathrm{B}$ beweist.

Tab. 1. ${ }^{1} \mathrm{H}$-Chemische Verschiebungen ( $\delta$-Werte) und Kopplungskonstanten (Absolutwerte in $\mathrm{Hz}$ ) von Benzobenzvalenen in Deuterochloroform mit Tetramethylsilan als internem Standard; $b=$ sehr breit

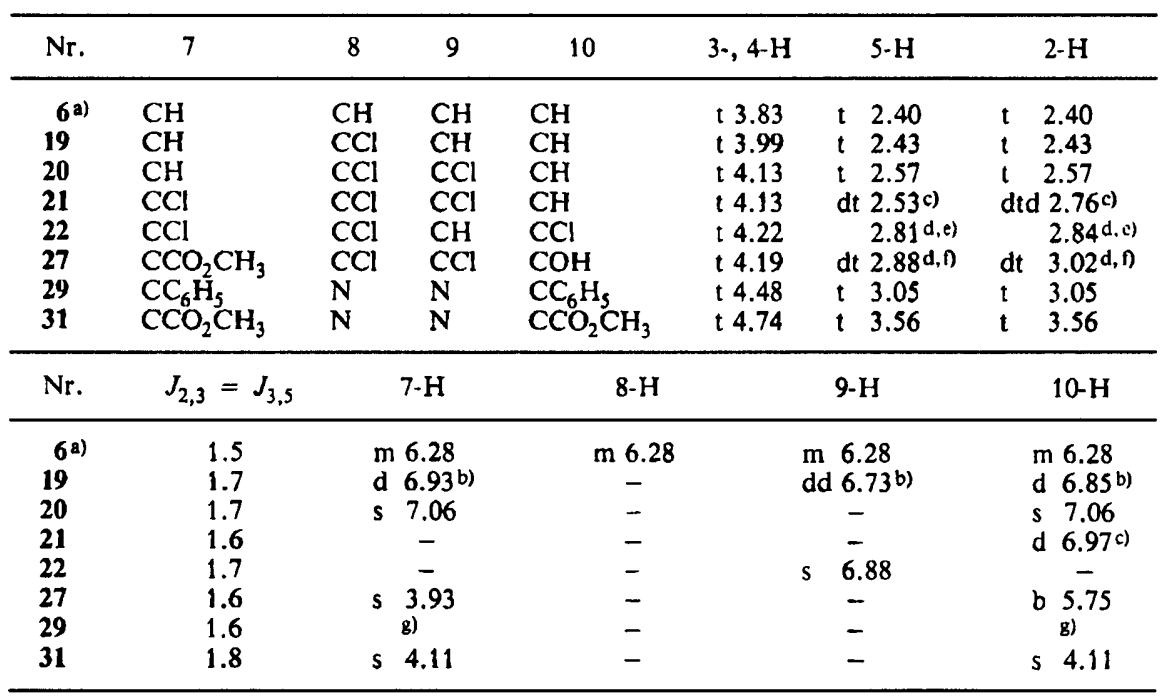

a) Aus Lit. ${ }^{16)}$, Lösungsmittel $\mathrm{CCl}_{4} .-$ b) $J_{7,9}=1.7, J_{9,10}=7.5 \mathrm{~Hz}$. c) $J_{2,5}=5.6, J_{2,10}=$ $0.7 \mathrm{~Hz}$. - d) Diese Zuordnungen sind austauschbar. - e) Diese chemische Verschiebungen sowie $J_{2,5}=5.7 \mathrm{~Hz}$ wurden dem Spektrum des $21 \mathrm{a}, 22 \mathrm{a}$-Gemisches entnommen. - $f J_{2.5}=5.4 \mathrm{~Hz}$. 8) $\mathrm{m} 7.30-7.66(6 \mathrm{H}), \mathrm{m} 7.83-8.06(4 \mathrm{H})$.

Das gleiche Phănomen beobachtet man in den ${ }^{13} \mathrm{C}$-NMR-Spektren (Tab. 2). Hier

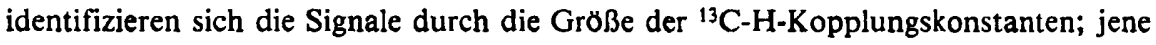
von C-3,4 $(\delta=42.4-48.6)$ weisen Werte von $213.3-222.5 \mathrm{~Hz}$ auf und die von C-2 und $\mathrm{C}-5(\delta=36.4-39.2)$ nur solche von $170.2-184.8 \mathrm{~Hz}$. Uber die direkte $\mathrm{C}-\mathrm{H}$ Kopplung hinaus geben die protonengekoppelten Spektren auch weiterreichende Wechselwirkungen preis. Die Feinstruktur der C-3,4-Signale - ein Dublett von 4- $5 \mathrm{~Hz}$ - kann aus Symmetriegrunden nur von $J_{\mathrm{C}-3,4-\mathrm{H}}$ stammen, woraus folgt, daß $J_{\mathrm{C}-3,2-\mathrm{H}}$ und $J_{\mathrm{C}-3,5-\mathrm{H}}$ kleiner als die Auflösung sein müssen. Umgekehrt haben $J_{\mathrm{C}-2,3-\mathrm{H}}$ und $J_{\mathrm{C}-5,3-\mathrm{H}}$ mit ca. $3.5 \mathrm{~Hz}$ durchaus meßbare Werte. Eine zusătzliche Aufspaltung gleicher 


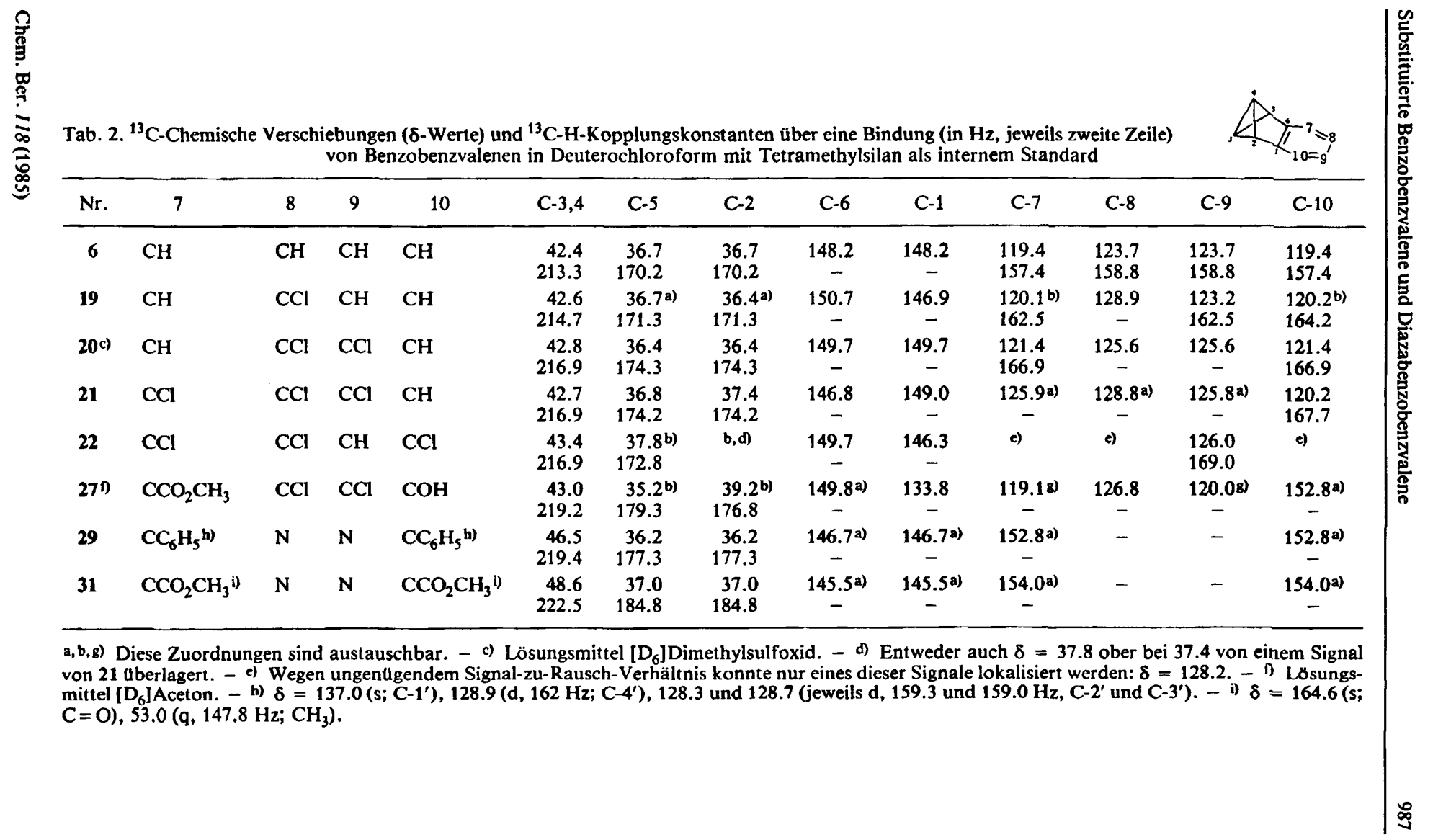


Großße tritt in den C-2- und C-5-Banden auf, wenn die Gruppen 7 bzw. 10 ein H-Atom tragen. Diese Kopplungen $\left(J_{\mathrm{C}-5,7-\mathrm{H}}\right.$ und $\left.J_{\mathrm{C}-2,10-\mathrm{H}}\right)$ beweisen die Positionen der Wasserstoff- und damit auch die der Chloratome in 20 und 21 und erlauben die spezifische $\mathrm{Zu}$ ordnung der C-2- und C-5-Signale in 21. Die Vicinalkopplungen $J_{\mathrm{C}-2,5-\mathrm{H}}$ und $J_{\mathrm{C}-5,2-\mathrm{H}}$ werden durch zwei Bindungswege vermittelt, was vermutlich die Ursache für die recht großen Werte von $10.6-12.3 \mathrm{~Hz}$ ist.

Ebenfalls auf die Feinstrukturen stützt sich die Zuordnung der Aromaten-C-Resonanzen im unsubstituierten Benzobenzvalen (6). Ausgehend von den ${ }^{13} \mathrm{C}-\mathrm{H}-\mathrm{Kopplungs}$ konstanten in Benzol ${ }^{26 a, 27)}$ und Naphthalin ${ }^{26 b, 27)}$ hat man als aufgeloste weitreichende Wechselwirkungen für C-8 nur jene mit 10-H zu erwarten. Dementsprechend weisen wir das Signal mit der 6.3-Hz-Dublettaufspaltung $(\delta=123.7) \mathrm{C}-8,-9 \mathrm{zu}$. Andererseits sollte $\mathrm{C}-7$ sowohl mit 9-H als auch mit 5-H jeweils über drei Bindungen meßbar koppeln. Die Absorption bei $\delta=119.4$ zeigt denn auch die Doppeldublettfeinstruktur mit der wahrscheinlichen Zuordnung $J_{\mathrm{C}-7,9-\mathrm{H}}=6 \mathrm{~Hz}$ und $J_{\mathrm{C}-7,5-\mathrm{H}}=3.5 \mathrm{~Hz}$. Weitere Spezifizierungen hinsichtlich der aromatischen Signale beruhen auf dem Einfluß der jeweiligen Substituenten in monosubstituierten Benzolen ${ }^{27}$.

Den in der Einleitung diskutierten besonderen Tieffeldeffekt in den Spektren von Benzvalen (4) und anderen Verbindungen führen wir auf die Wechselwirkung zwischen einem besetzten Orbital des gespannten $\sigma$-Systems und dem $\pi^{*}$-Orbital des ungesăttigten Molekülteils zurück ${ }^{2)}$. Wie bei 4 ubernimmt bei den Benzobenzvalenen das $a_{2}$-Orbital des Bicyclo[1.1.0]butan-Systems (33) die Rolle des Donors, während als Akzeptororbital des aromatischen $\pi$-Systems nur $e_{2 u}(34)$ in Frage kommt.

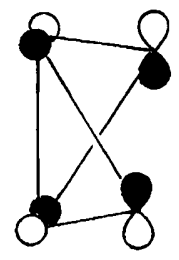

33

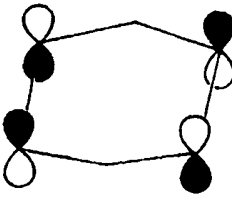

36

Bezogen auf die gemeinsame Referenz Tricyclo[3.1.0.0 2,6 $]$ hexan (5) ist der Tieffeldeffekt im Benzobenzvalen (6) um 5.9 ppm kleiner als im Benzvalen (4). Dieser Befund laßt sich mit dem Orbitalwechselwirkungsmodell interpretieren. Das Ausmaß einer derartigen Interaktion hängt von der Energiedifferenz der Orbitale und deren Koeffizientengröße an den wechselwirkenden Zentren ab. Zwar ist nach HMO die Energie des Benzol- $e_{2 u}$-Orbitals (34) mit $-1.00 \beta$ gleich derjenigen des Ethylen- $\pi *$-Niveaus, jedoch betragen die Koeffizienten von Belang nur 0.500 gegenuber $0.707^{28}$. Diese Vorstellungen fordern, daß die chemischen Verschiebungen der Positionen 3,4 durch Substituenten am oder Heteroatome im aromatischen Ring der Benzobenzvalene beeinflußbar sein sollten. Die Tabellen 1 und 2 bestätigen dies. Wenn auch der Effekt der Chloratome in 19-22 gering ist, so uben die Stickstof fatome und Substituenten in 29 und 31 doch deutliche Verănderungen nach tiefem Feld aus: bezogen auf 60.65 bzw. $0.91 \mathrm{ppm}$ in den ${ }^{1} \mathrm{H}$ - und $4.1 \mathrm{bzw}, 6.2 \mathrm{ppm}$ in den ${ }^{13} \mathrm{C}$-NMR-Spektren. Dies steht im Einklang mit dem Modell, denn die Stickstoffatome in 29 und 31 und darüber hinaus die Carbon- 
săureester-Substituenten in $\mathbf{3 1}$ stabilisieren 34 und verbessern so die Wechselwirkung mit 33. Im tetrasubstituierten Homocyclus von 27 kompensieren offenbar Carbonsăureester und Hydroxylfunktion weitgehend ihre Wirkung.

Diskutiert man chemische Verschiebungen von Bicyclo[1.1.0]butan-Bruckenkopfpositionen, darf man einen weiteren Parameter nicht außer acht lassen, nămlich den Winkel zwischen den beiden Dreiringen. So waren wir durch die Spektren des Phenalenabkömmlings 35 zunächst uberrascht, weil die Bicyclo[1.1.0]butan-Brückenkopf$\mathrm{CH}$-Gruppen praktisch normale chemische Verschiebungen zeigen. Ein besonderer Tieffeldeffekt tritt hier also nicht auf, obwohl Naphthalin im $b_{3 \mathrm{~g}}$-Orbital (36) uber einen Akzeptor geeigneter Symmetrie und besonders günstiger Energie $\left(-0.618 \beta^{28)}\right)$ verfugt und dessen Koeffizienten an den mit dem Bicyclo[1.1.0]butan-System wechsel-

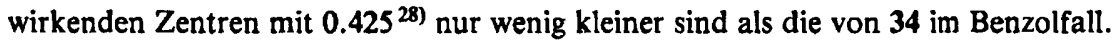

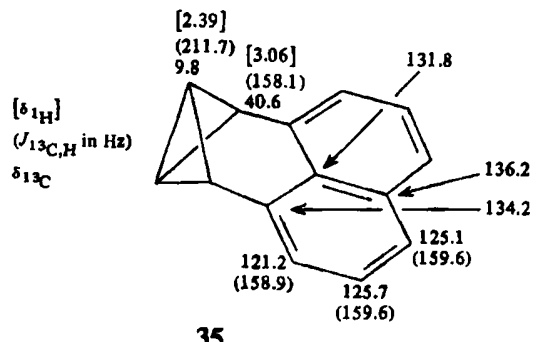

35
36

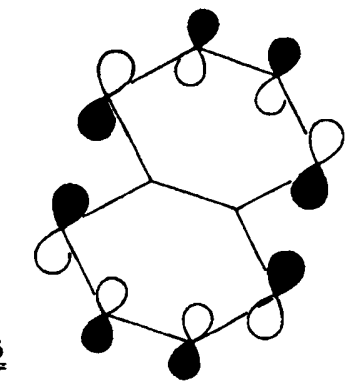

Entscheidend scheint hier die Änderung einer Eigenschaft des Bicyclo[1.1.0]butanSystems zu sein, die mit der Variation des Interplanarwinkels einhergeht. Die Abhängigkeit der Orbitalenergien von diesem Parameter haben zuerst Gleiter et al. ${ }^{29 a}$ untersucht. Spătere Arbeiten bestătigen die Ergebnisse ${ }^{30}$, denen zufolge sich das $a_{2}$-Orbital 33 mit größer werdendem Interplanarwinkel beträchtlich stabilisiert. Im Rahmen unseres Modells kann es dann weniger gut mit einem Akzeptororbital wechselwirken. Dies dürfte in 35 zutreffen, wo wegen der Dreikohlenstoffbrücke über dem Bicyclo[1.1.0]butan-System dieser Winkel ${ }^{29 b)}$ mit ca. $119^{\circ}$ gegenüber ca. $108^{\circ}$ in 6 erheblich aufgeweitet ist.

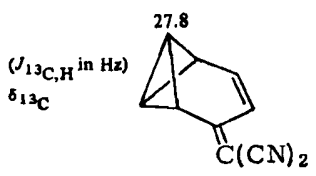

37<smiles>O=C1C=CC2C3C1C23</smiles>

38

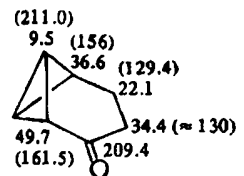

39<smiles>OC1C=CC2C3C1C23</smiles>

40

Wie die Verbindungen 37, 38 und $\mathbf{4 0}$ zeigen, beobachtet man die diskutierten Tieffeldeffekte auch im Falle von Dreikohlenstoffbrucken, wenn sie über ein genügend günstiges Akzeptororbital verfügen. An den Anknüpfungspunkten des Bicyclo[1.1.0]butan-Systems haben die $\pi^{*}$-Orbitale von 1,3-Butadien- und Acrolein-Einheit unterschiedliche Koeffizientenvorzeichen, so daß die Wechselwirkung mit dem $a_{2}-$ Orbital 33 möglich ist. In 37 sorgen die beiden Nitrilgruppen für die relativ niedrige Energie des 
$\pi^{*}$-Orbitals und in 38 das Sauerstoffatom mit der Konsequenz, daß die Bicyclo[1.1.0]butan-Brückenkopf-C-Atome bei ungewöhnlich tiefem Feld $\left(\delta=27.8^{31}\right)$ bzw. 29.932,33) ) absorbieren. Das gesăttigte Keton 39 bezeugt die Wirkungslosigkeit der Carbonylfunktion, wenn nicht die beidseitige Konjugationsfähigkeit der Brücke gegeben ist. Gegenüber dem Stammkohlenwasserstoff Tricyclo[4.1.0.0 $0^{2,7}$ heptan $(\delta=$ 5.9 ${ }^{34)}$ ) zeigen $\mathrm{C}-1,-7$ in 39 eine Veränderung um nur $3.6 \mathrm{ppm}$ nach tiefem Feld. Eine drastische Steigerung der Akzeptorqualităt trìtt durch die Protonierung des Carbonylsauerstoffs in $38 \mathrm{zu} 40 \mathrm{ein}$, und die C-1, -7-Absorption erreicht mit $\delta=75.5^{33)}$ die Rekordmarke.

Der Hochfeldextrempunkt wird gegenwärtig vom Octavalen (41) mit $\delta=-13.4$ gehalten 35a). Wir betrachten dies als Beleg der Unwirksamkeit der 1,3-Butadienbrücke, weil sie mil den Positionen 1 und 4 angeknüpft ist, wo die $\pi^{*}$-Orbital-Koeffizienten gleiches Vorzeichen haben und daher die Wechselwirkung mit dem $a_{2}$-Orbital 33 verhindern. Allerdings verursacht hier der große Interplanarwinkel (ca. $130^{\circ}$ ) eine extreme Stabilisierung von 33, womit in Analogie zu 35 selbst bei geeigneter Symmetrie ein bedeutender Tieffeldeffekt nicht zu erwarten wäre. Auch in 42 läßt die unterschiedliche Symmetrie des $\pi^{*}$-Orbitals der 1,3-Butadienbrücke die Wechselwirkung mil dem $a_{2}$-Orbital 33 des $\sigma$-Systems nicht zu. Die chemische Verschiebung $\delta=11.735$ b) liegt in Übereinstimmung damit kaum außerhalb des Bereichs normaler Werte. Tricyclo[2.1.0.0 $0^{2,5}$ pentan3-on (43) verfügt wegen des sehr kleinen Interplanarwinkels über ein höchst energiereiches $\mathrm{a}_{2}$-Orbital 33, und das energiearme $\pi^{*}$-Orbital der Carbonylgruppe wäre ein vorzüglicher Akzeptor, wenn die unterschiedliche Symmetrie die Wechselwirkung nicht verböte. Mit $\delta=-1.0^{36}$ ) weicht die chemische Verschiebung tatsăchlich nur um 2 ppm von der des unsubstituicrten Bicyclo[1.1.0]butans $\left(\delta=-3^{37}\right)$ ab.

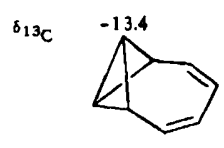

41

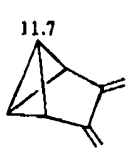

42

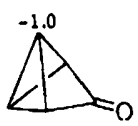

43

Günstige Akzeptororbitale stellen nicht nur $\pi$-Systeme bereit, sondern auch Dreiringe ${ }^{5,38)}$. Wirkungsvolle besetzte Orbitale kann man bisher außer dem 2,4-überbrückten Bicyclo[1.1.0]butan-System, wo die größten Effekte auftreten, nur noch 1,3-überbrückten Cyclobutan- und Cyclopentanringen zuschreiben. Mit abnehmender Spannung der betreffenden $\sigma$-Bindungen werden die ${ }^{13} \mathrm{C}$-NMR-Effekte kleiner.

$\mathrm{Daß}$ über die beschriebene Wechselwirkung hinaus andere Faktoren die chemischen Verschiebungen beeinflussen, machen $\mathbf{4 1}$ und $\mathbf{4 2}$ mit einem Unterschied von $25.1 \mathrm{ppm}$ deutlich. Möglicherweise ließe sich das grobe qualitative Modell verfeinern, etwa durch Einbeziehung weiterer besetzter und unbesetzter Orbitale. Dazu besteht jedoch kein Anlaß, weil bisher zwischen den NMR-Effekten und Moleküleigenschaften - obige Orbitalwechselwirkung generiert ja zwei neue Molekülorbitale und daraus resultierend eine neue Ladungsverteilung - eine Korrelation nicht hergestellt werden kann. Mit Hilfe einer von Schindler und Kutzelnigg ${ }^{39)}$ entwickelten neuen Theorie sollte die Berechnung der chemischen Verschiebung der diskutierten Moleküle mit hinreichender Genauigkeit und ihre Zerlegung in Orbitalbeiträge möglich sein, so daß eine Prüfung des qualitativen Modells in Sicht ist.

S. F. dankt dem Verband der Chemischen Industrie e. V. für ein Chemiefonds-Stipendium. Auch der Deutschen Forschungsgemeinschaft verdanken wir finanzielle Förderung. Herr Dr. D. Wendisch, Bayer AG, Leverkusen, ubernahm dankenswerterweise die Messung der NMR-

Chem. Ber. 118 (1985) 
Spektren von 10 einschließlich der Entkopplungsexperimente. Unser Dank gilt ferner der Union Rheinische Braunkohlen Kraftstoff AG, Wesseling, für Dimethylether-Spenden.

\title{
Experimenteller Teil
}

'H-NMR: Varian EM 390; Bruker WH 360, WM 400. - ${ }^{13} \mathrm{C}$-NMR: Bruker WH 90, WH 360, WM 400. - MS: Varian MAT CH 7. - IR: Beckman AccuLab 4. - UV: Beckman DB-GT. Elementaranalysen: Perkin-Elmer 240 Elemental Analyzcr, Carlo Erba Strumentatione Elemental Analyzer Mod. 1106.

\section{A. Diels-Alder-Addukte des Benzvalens und ihre Umwandiung in Tetracyclo[4.4.0.0 $\left.0^{2,4} \cdot 0^{3,5}\right]$ deca-7,9-diene}

1,12-Dichlor-9-oxapentacyclol6.2.2.0 $0^{2,7} \cdot 0^{3,5} \cdot 0^{4.6} \mathrm{Jdodec}-11$-en-10-on (8): $1.22 \mathrm{~g}(7.39 \mathrm{mmol})$ 3,5-Dichlor- $\alpha$-pyron ${ }^{40)}$ wurden in $20 \mathrm{ml}(8.92 \mathrm{mmol})$ etherischem Benzvalen $(4)^{16,41,42)}$ gelost und $24 \mathrm{~h}$ bei Raumtemp. belassen. Danach dampfte man den Ether i. Vak. ab, wobei ein braunes Öl zurückblieb, das beim Anreiben mit Ether kristallisierte. Die Tieftemperaturkristallisation aus Ether erbrachte $1.02 \mathrm{~g}(56 \%) 8$ als farblose Kristalle mit Schmp. $97-99^{\circ} \mathrm{C}$. - IR (KBr): 3090 (m), 3041 (w), 2927 (m) (jeweils C - H); 1779 (s), 1767 (s) (jeweils C=O); $1644(\mathrm{~m}), 1633 \mathrm{~cm}^{-1}$ (m) (jeweils $C=C)$. - UV ( $n$-Hexan): $\lambda_{\max }(\lg \varepsilon)=233 \mathrm{~nm}(3.16) .-M S(70 \mathrm{eV}): m / e=244$, $242\left(0.02 \%, 0.04 \%, \mathrm{M}^{+}\right), 165,163\left(11,34, \mathrm{C}_{10} \mathrm{H}_{8} \mathrm{Cl}\right), 136(14), 128$ (49), $127(39), 115(14), 78$ (31), $63(16), 52\left(100, \mathrm{C}_{4} \mathrm{H}_{4}\right), 51(19) .-{ }^{1} \mathrm{H}-\mathrm{NMR}\left(\mathrm{CDCl}_{3}\right): \delta=2.00-2.38(\mathrm{~m} ; 3-, 4-, 5-, 6-\mathrm{H})$, 2.53 (dq, $J_{2,3}=J_{2,5}=J_{2,11}=1.1, J_{2,7}=8.0 \mathrm{~Hz} ; 2-\mathrm{H}$ ), 2.90 (br. dd, $J_{7,8}=4.2 \mathrm{~Hz} ; 7-\mathrm{H}$ ), 4.88 (dd, $\left.J_{8,11}=1.2 \mathrm{~Hz} ; 8-\mathrm{H}\right), 6.13(\mathrm{dd} ; 11-\mathrm{H}) .-{ }^{13} \mathrm{C}$-NMR $\left(\mathrm{CDCl}_{3}\right): \delta=2.3$ und $3.7(\mathrm{C}-4,-5), 34.0$ und 34.1 (C-3, -6), 47.8 und 48.6 (C-2, -7), 68.8 (C-1), 80.9 (C-8), 127.9 (C-11), 130.5 (C-12), $167.9(C-10)$

\author{
$\mathrm{C}_{11} \mathrm{H}_{8} \mathrm{Cl}_{2} \mathrm{O}_{2}$ (243.1) Ber. C 54.35 H 3.32 Gef. C 54.17 H 3.41
}

1,11,12-Trichlor-9-oxapentacyclo/6.2.2.0 $0^{2,7} \cdot 0^{3,5} \cdot 0^{4,6} \mathrm{Jdodec}-11$-en-10-on (9): $5.60 \mathrm{~g} \quad$ (28.1 mmol) 3,4,5-Trichlor- $\alpha$-pyron ${ }^{8)}$ wurden bei Raumtemp. $4 \mathrm{~h}$ in $70 \mathrm{ml}$ (31.2 mmol) etherischem 4 gerührt. Der ausgefallene, farblose Niederschlag wurde abgesaugt, mit kaltem Ether gewaschen und getrocknet $(3.69 \mathrm{~g})$. Aus der $\mathrm{i}$. Vak, eingeengten Mutterlauge wurden weitere $4.00 \mathrm{~g} 9$ isoliert. Nach der Tieftemperaturkristallisation aus Ether erhielt man insgesamt $7.43 \mathrm{~g}(95 \%) 9$ als farblose Kristalle mit Schmp. $128-130^{\circ} \mathrm{C}$. - IR (KBr): 3145 (w), 3060 (w), 3015 (w), 2942 (w) (jeweils $\mathrm{C}-\mathrm{H}) ; 1780(\mathrm{~s}, \mathrm{C}=\mathrm{O}), 1630 \mathrm{~cm}^{-1}(\mathrm{~m}, \mathrm{C}=\mathrm{C})$. - UV (n-Hexan): $\lambda_{\max }(\lg \varepsilon)=235(3.30)$, $208 \mathrm{~nm}(3.56)$. - MS $(70 \mathrm{eV}): m / e=280,278,276\left(0.02 \%, 0.07 \%, 0.07 \%, \mathrm{M}^{+}\right), 201,199,197$ $\left(2,12,18, \mathrm{C}_{10} \mathrm{H}_{7} \mathrm{Cl}_{2}\right), 164,162\left(12,39, \mathrm{C}_{10} \mathrm{H}_{7} \mathrm{Cl}\right), 161(10), 78\left(39, \mathrm{C}_{6} \mathrm{H}_{6}\right), 63(10), 52\left(100, \mathrm{C}_{4} \mathrm{H}_{4}\right)$, 51 (16). - ' ${ }^{1} \mathrm{H}-\mathrm{NMR}\left(\mathrm{CDCl}_{3}\right): \delta=2.00-2.38(\mathrm{~m} ; 3-, 4-, 5-, 6-\mathrm{H}), 2.60\left(\mathrm{dt}, J_{2,7}=7.5, J_{2,5}=\right.$ $\left.J_{2,3}=1.2 \mathrm{~Hz} ; 2-\mathrm{H}\right), 2.88$ (br. dd, $\left.J_{7,8}=4.2 \mathrm{~Hz} ; 7-\mathrm{H}\right), 5.03(\mathrm{~d} ; 8-\mathrm{H}) .-{ }^{13} \mathrm{C}-\mathrm{NMR}\left(\mathrm{CDCl}_{3}\right): \delta=$ 2.3 und $2.4(\mathrm{C}-4,-5), 33.7$ und $34.2(\mathrm{C}-3,-6), 47.5$ und $49.2(\mathrm{C}-2,-7), 72.6(\mathrm{C}-1), 80.3(\mathrm{C}-8), 126.0$ und $127.2(\mathrm{C}-11,-12), 166.5(\mathrm{C}-10)$.

$$
\mathrm{C}_{11} \mathrm{H}_{7} \mathrm{Cl}_{3} \mathrm{O}_{2} \text { (277.5) Ber. C } 47.61 \mathrm{H} 2.54 \text { Gef. C } 47.76 \mathrm{H} 2.74
$$

1,8,11,12-Tetrachlor-9-oxapentacyclo[6.2.2.0 $\left.2,7.0^{3,5} \cdot 0^{4,6}\right]$ dodec-11-en-10-on (10): $7.40 \mathrm{~g}$ (31.6 mmol) Tetrachlor- $\alpha$-pyron ${ }^{40)}$ rührte man bei Raumtemp. $3 \mathrm{~h}$ in $75 \mathrm{ml}(32.1 \mathrm{mmol})$ etherischem 4. Danach engte man $i$. Rotationsverdampfer ein, wobei $8.52 \mathrm{~g}(86 \%)$ hellgelbe Kristalle zurilckblieben. Tieftemperaturkristallisation aus Ether fuhrte zu $8.12 \mathrm{~g} \mathrm{(82 \% )} 10 \mathrm{in}$ Form farbloser Kristalle mit Schmp. $76-78^{\circ} \mathrm{C}$. - IR (KBr): $3130(\mathrm{~m}), 3115(\mathrm{~m}), 3070(\mathrm{w}), 2950$ (w) (jeweils C - H); 1795 $(\mathrm{s}, \mathrm{C}=\mathrm{O}), 1605 \mathrm{~cm}^{-1}(\mathrm{~m}, \mathrm{C}=\mathrm{C}) .-\mathrm{MS}(70 \mathrm{eV})$ : Identisch mit MS von 13 $\left.{ }^{4 \mathrm{a}}\right)$, dem Thermolyseprodukt von 10. $-{ }^{1} \mathrm{H}-\mathrm{NMR}\left(\mathrm{CDCl}_{3}\right): \delta=3.18\left(\mathrm{dt}, J_{2,7}=8.0, J_{5,7}=J_{6,7}=1.0 \mathrm{~Hz} ; 7-\mathrm{H}\right), 2.68$ $\left(\mathrm{dt}, J_{2,3}=J_{2,5}=1.0 \mathrm{~Hz} ; 2-\mathrm{H}\right), 2.32\left(\mathrm{dm}, J_{4,5}=9.0, J_{3,5}=J_{5,6}=1.7 \mathrm{~Hz} ; 5-\mathrm{H}\right), 2.27\left(\mathrm{dm}, J_{3,6} \approx\right.$ $5 \mathrm{~Hz} ; 3-\mathrm{H}), 2.26\left(\approx \mathrm{dt}, J_{3,4}=J_{4,6}=2.0 \mathrm{~Hz} ; 4-\mathrm{H}\right), 2.20(\mathrm{~m}, 6-\mathrm{H})$. Die Zuordnung wurde auf- 
grund von Entkopplungsexperimenten getroffen unter der Annahme, daß in Ubereinstimmung mit den Verhältnissen in 8 und 9 das Signal bei $\delta=3.18$ von $7-\mathrm{H}$ stammt. $-{ }^{13} \mathrm{C}-\mathrm{NMR}\left(\mathrm{CDCl}_{3}\right)$ : $\delta=2.1$ und 2.6 (jeweils $\mathrm{d}, 216 \mathrm{~Hz} ; \mathrm{C}-4,-5$ ), 33.9 und 34.8 (jeweils $\mathrm{d}, 165 \mathrm{~Hz} ; \mathrm{C}-3,-6$ ), 50.4 und 57.2 (jeweils d, $147 \mathrm{~Hz} ; \mathrm{C}-2,-7), 71.9$ (s; C-1), 99.3 (s; C-8), 128.0 und 128.1 (jeweils s; C-11, -12), 163.6 (s; C-10).

$\mathrm{C}_{11} \mathrm{H}_{6} \mathrm{Cl}_{4} \mathrm{O}_{2}$ (312.0) Ber. C $42.35 \mathrm{H} 1.93 \mathrm{Cl} 45.45$ Gef. C $42.06 \mathrm{H} 2.03 \mathrm{Cl} 45.53$

7,9-Dichlortetracyclo[4.4.0.02,4.03.5 Jeca-7,9-dien (11): $1.00 \mathrm{~g}$ (4.11 mmol) 8 wurden $10 \mathrm{~min}$ lang auf $148^{\circ} \mathrm{C}$ (nicht höher!) erhitzt. Nach dem Erkalten nahm man das braune Thermolyseprodukt in $n$-Hexan auf und filtrierte es uber basisches $\mathrm{Al}_{2} \mathrm{O}_{3}$ (Akt.-Stufe III). Abziehen des Hexans im Rotationsverdampfer hinterließ ein braunes Öl, das bei $50^{\circ} \mathrm{C}(\mathrm{Bad}) / 0.1$ Torr destilliert wurde. Es gingen $570 \mathrm{mg}(69 \%) 11$ als blaues Ol über. Bei Raumtemp. zersetzte sich 11 innerhalb von $48 \mathrm{~h}$. Wegen geringfügiger Verunreinigungen konnte keine korrekte Kohlenstoffanalyse erhalten werden. - IR (Film): 3140 (w), 3045 (w), 2910 (w) (jeweils C - H); 1643 (s), $1604 \mathrm{~cm}^{-1}(\mathrm{~m})$ (jeweils $C=C)$. MS $(70 \mathrm{eV}): m / e=202,200,198\left(0.3 \%, 2 \%, 3 \%, \mathrm{M}^{+}\right), 163(9), 128(14), 127$ (12), $52\left(100, \mathrm{C}_{4} \mathrm{H}_{4}\right)$. - ' $\mathrm{H}-\mathrm{NMR}\left(\mathrm{CDCl}_{3}\right): \delta=1.90$ und 2.09 (jeweils dm, $J_{3,4}=9.6 \mathrm{~Hz}$; 3-, 4-H), 2.31 und 2.60 (jeweils dm, $J_{2,5}=5.1 \mathrm{~Hz} ; 2-, 5-\mathrm{H}$ ), 2.68 und 2.80 (jeweils dm, $J_{1,6}=$ $11.5 \mathrm{~Hz} ; 1-, 6-\mathrm{H}), 5.63(\mathrm{~m} ; 10-\mathrm{H}), 5.83$ (br. s; 8-H). $-{ }^{13} \mathrm{C}-\mathrm{NMR}\left(\mathrm{CDCl}_{3}\right): \delta=0.6$ und 2.4 $(C-3,-4), 40.9,42.3,43.3$ und $45.4(C-1,-2,-5,-6), 121.6$ und $121.9(C-8,10), 124.2(C-9), 137.5$ (C-7).

$$
\mathrm{C}_{10} \mathrm{H}_{8} \mathrm{Cl}_{2} \text { (199.1) Ber. } \mathrm{C} 60.32 \text { H } 4.05 \text { Gef. C } 58.94 \text { H } 4.29
$$

7,8,9-Trichlortetracyclo[4.4.0.02,4.03,5]deca-7,9-dien (12): $1.50 \mathrm{~g}(5.40 \mathrm{mmol}) 9$ wurden $10 \mathrm{~min}$ auf $150^{\circ} \mathrm{C}$ (nicht höher!) erhitzt. Das braune Thermolyseprodukt erstarrte beim Erkalten. Es wurde durch Filtration seiner $n$-Hexanlosung durch basisches $\mathrm{Al}_{2} \mathrm{O}_{3}$ (Akt.-Stufe III) teilweise entfärbt. Nach dem Abdampfen des Hexans i. Vak. blieben $730 \mathrm{mg}$ (57\%) 12 als grünes Öl zurück, das im Kühlschrank erstarrte, Schmp. $31-32^{\circ} \mathrm{C}$. - IR (Film): 3138 (w), 3050 (w), 2910 (w) (jeweils $C-H) ; 1626(\mathrm{~m}), 1588 \mathrm{~cm}^{-1}(\mathrm{~m})$ (jeweils $\left.\mathrm{C}=\mathrm{C}\right)$. $-\mathrm{MS}(70 \mathrm{eV}): m / e=236,234,232(1 \%$, $\left.5 \%, 7 \%, \mathrm{M}^{+}\right), 197(14), 162(27), 52\left(100, \mathrm{C}_{4} \mathrm{H}_{4}\right) .-\mathrm{UV}\left(n\right.$-Hexan): $\lambda_{\max }(\lg \varepsilon)=302(3.28), 290$ (3.52), 279 (3.51), 269 (3.40), $230 \mathrm{~nm}$ (3.62). $-{ }^{1} \mathrm{H}-\mathrm{NMR}\left(\mathrm{CDCl}_{3}\right): \delta=1.93$ und 2.13 (jeweils dtt, $J_{3,4}=9.0 \mathrm{~Hz}$, Kopplungen zu 2-H und $5-\mathrm{H} 1.8 \mathrm{~Hz}$, Kopplungen $\mathrm{zu} 1-\mathrm{H}$ und $\left.6-\mathrm{H} 0.9 \mathrm{~Hz} ; 3-, 4-\mathrm{H}\right)$, $2.36\left(\mathrm{dm}\right.$, bei Entkopplung bei $\delta=5.86 \mathrm{dtd}, J_{2,5}=5.4, J_{1,2}=0.9 \mathrm{~Hz} ; 2-\mathrm{H}$ ), 2.63 (wegen Überlagerung des Hochfeldastes von 1- $\mathrm{H} \mathrm{m}$, als dtd interpretierbar, $\left.J_{5,6}=0.9 \mathrm{~Hz} ; 5-\mathrm{H}\right), 2.77(\mathrm{dm}$, bei Entkopplung bei $\delta=5.86$ als dq interpretierbar, $\left.J_{1.6}=11.4 \mathrm{~Hz} ; 1-\mathrm{H}\right), 2.87$ (dq; 6-H), 5.86 (dd, $\left.J_{1,10}=3.7, J_{2.10}=0.6 \mathrm{~Hz} ; 10-\mathrm{H}\right) . \quad{ }^{13} \mathrm{C}-\mathrm{NMR}\left(\mathrm{CDCl}_{3}\right): \delta=0.9$ und $2.6(\mathrm{C}-3,-4), 41.7,42.0$ und $42.5(\mathrm{C}-2,-5,-1), 47.6(\mathrm{C}-6), 123.1$ und $124.8(\mathrm{C}-8,-9), 126.3(\mathrm{C}-10), 133.6(\mathrm{C}-7)$.

$$
\mathrm{C}_{10} \mathrm{H}_{7} \mathrm{Cl}_{3} \text { (233.5) Ber. C } 51.43 \text { H } 3.02 \text { Gef. C } 51.72 \text { H } 3.32
$$

7,8,9,10-Tetrachlortetracyclo[4.4.0.0 $2,4 \cdot 0^{3,5}$ Jdeca-7,9-dien (13)

a) Durch Thermolyse von 10: $1.00 \mathrm{~g}(3.20 \mathrm{mmol}) 10$ wurden in $7 \mathrm{ml}$ frisch destilliertem Nitrobenzol gelöst und $20 \mathrm{~min}$ auf $150^{\circ} \mathrm{C}$ erhitzt. Die Lösung färbte sich tiefschwarz. Nach dem Abdestillieren des Nitrobenzols verblieben $840 \mathrm{mg}(\mathbf{9 7 \%})$ brauner Ruckstand, der sich ${ }^{1} \mathrm{H}-\mathrm{NMR}$-spektroskopisch als praktisch reines $13^{4 a)}$ erwies.

b) Aus Tetrachlorthiophen-1,1-dioxid und 4: Der Vereinigung von $1.00 \mathrm{~g}$ (3.94 mmol) Tetrachlorthiophen-1,1-dioxid ${ }^{13)}$ mit $10 \mathrm{ml}(3.95 \mathrm{mmol})$ etherischem 4 folgte eine heftige $\mathrm{SO}_{2}$-Entwicklung. Nach deren Abklingen engte man im Rotationsverdampfer ein und erhielt $1.05 \mathrm{~g} \mathrm{(99 \% )}$ reines 13, identisch mit einer authentischen Probe $\left.{ }^{4 a}\right)$.

\section{B. Photolyse und Diels-Alder-Addukte der Tetracyclo[4.4.0.0 $\left.2.4 .0^{3,5}\right]$ deca-7,9-diene}

exo- (14a) und endo-Pentacyclo/4.4.0.0 $0^{2,4} \cdot 0^{3,5} \cdot 0^{7,10}$ Jdec-8-en (14 b): $290 \mathrm{mg}(2.22 \mathrm{mmol}) 7$ wurden bei $20^{\circ} \mathrm{C}$ in $250 \mathrm{ml}$ absol. Ether nach Sättigen mit $\mathrm{N}_{2}$ durch Quarz solange (einige h) mit

Chem. Ber. 118 (1985) 
einer $\mathrm{Hg}$-Niederdrucklampe (Gräntzel-Reaktor 400) belichtet, bis das UV-Spektrum der Lösung den vollständigen Verbrauch von 7 anzeigte. Man entfernte den Ether im Rotationsverdampfer. Aus dem Rückstand destillierten bei $50-70^{\circ} \mathrm{C}$ (Bad)/12 Torr $190 \mathrm{mg}(65 \%)$ 14a und $14 \mathrm{~b}$ als farblose Flüssigkeit. Aufgrund von Lösungsmittelresten wurden korrekte Elementaranalysenwerte nicht erzielt. - IR (Film): 3025 (s), 2937 (s) (jeweils C-H); 775 (s), 747 (s), $720 \mathrm{~cm}^{-1}$ (s). - MS $(70 \mathrm{eV}): m / e=130\left(10 \%, \mathrm{M}^{+}\right), 129(43), 128(41), 115(33), 52\left(100, \mathrm{C}_{4} \mathrm{H}_{4}\right) .-{ }^{1} \mathrm{H}-$ NMR $\left(\mathrm{CDCl}_{3}\right.$; nur die Banden des Hauptisomeren $14 \mathrm{a}$ sind angegeben, da jene von $14 \mathrm{~b}$ wegen Uberlagerung nicht sicher lokalisiert werden konnten; alle Banden erscheinen jeweils als enges Multiplett): $\delta=1.90(2 \mathrm{H}), 2.07(2 \mathrm{H}), 2.27(2 \mathrm{H}), 2.82(2 \mathrm{H}), 6.21(8-, 9-\mathrm{H}) . \quad-{ }^{13} \mathrm{C}-\mathrm{NMR}$ $\left(\mathrm{CDCl}_{3}\right.$; die Bandenhöhen ergeben das Isomerenverhältnis 14a:14b $=84: 16$; die Unterscheidung der Signale von C-3 und C-4 gelingt aufgrund des bekannten Feinstrukturkriteriums $\mathrm{s}^{4 a}, 11 \mathrm{~b}$ ): 14a: $\delta=-1.1(\mathrm{~d}, 214 \mathrm{~Hz} ; \mathrm{C}-4), 6.8(\mathrm{~d}, 213 \mathrm{~Hz} ; \mathrm{C}-3), 35.6(\mathrm{~d}, \approx 165 \mathrm{~Hz} ; \mathrm{C}-2,-5), 44.8$ und 47.5 (d, 144 bzw. $142 \mathrm{~Hz}$; C-1, -6 und C-7, -10), 140.8 (d, $168 \mathrm{~Hz} ; \mathrm{C}-8,-9) .14 \mathrm{~b}: \delta=1.2$ (d, $212 \mathrm{~Hz}$; C-4), $5.8(\mathrm{~d}, 213 \mathrm{~Hz} ; \mathrm{C}-3), 35.0(\mathrm{~d}, \approx 160 \mathrm{~Hz} ; \mathrm{C}-2,-5), 37.2$ und $42.0(\mathrm{C}-1,-6$ und $\mathrm{C}-7,-10), 138.4$ (d, $167 \mathrm{~Hz} ; \mathrm{C}-8,-9)$.

7,8,9-Trichlor-exo- (15a) und -endo-pentacyclo[4.4.0.0 $2,4 \cdot 0^{3,5} \cdot 0^{7,10}$ ]dec-8-en (15b): $1.12 \mathrm{~g}$ $(4.80 \mathrm{mmol}) 12$ wurden bei $20^{\circ} \mathrm{C}$ in $150 \mathrm{ml}$ absol. Benzol und $5 \mathrm{ml}$ Triethylamin nach Săttigen mit $\mathrm{N}_{2} 10 \mathrm{~h}$ lang durch Pyrex mit einer Hg-Mitteldrucklampe belichtet (Hanovia $450 \mathrm{~W}$ ). Danach war 12 UV-spektroskopisch nicht mehr nachweisbar. Man filtrierte einen braunen Feststoff ab, engte das rotbraune Filtrat im Rotationsverdampfer ein, entfärbte das zurückbleibende Öl durch Filtration seiner $\mathrm{CHCl}_{3}$-Lösung durch basisches $\mathrm{Al}_{2} \mathrm{O}_{3}$ (Akt.-Stufe III) und engte wieder ein. Aus dem Ruckstand destillierten bei $60-65^{\circ} \mathrm{C}$ (Bad) $/ 0.001$ Torr $690 \mathrm{mg}(61 \%) 15 \mathrm{a}$ und $15 \mathrm{~b}$ als farblose Flüssigkeit, laut ${ }^{1}$ H-NMR-Spektrum im Verhăltnis 63: 37 , - IR (Film): 3140 (w), 3055 (m), 2990 (m), $2960(\mathrm{~m})$ (jeweils $\mathrm{C}-\mathrm{H}) ; 1615 \mathrm{~cm}^{-1}(\mathrm{~s})(\mathrm{C}=\mathrm{C}) .-\mathrm{MS}(70 \mathrm{eV}): \mathrm{m} / e=236,234,232(0.1 \%$, $\left.0.5 \%, 0.6 \%, \mathrm{M}^{+}\right), 162(26), 156(14), 154(14), 126(10), 52\left(100, \mathrm{C}_{4} \mathrm{H}_{4}\right) .-{ }^{1} \mathrm{H}-\mathrm{NMR}\left(\mathrm{CDCl}_{3}\right)$ : $15 \mathrm{a}: \delta=2.13-2.21(\mathrm{~m} ; 2 \mathrm{H}), 2.21-2.28(\mathrm{~m} ; 2 \mathrm{H}), 2.54\left(\mathrm{br}, \mathrm{d}, J_{2,5}=5.5 \mathrm{~Hz} ; 2\right.$ - oder $\left.5-\mathrm{H}\right)$, $2.78-2.83(\mathrm{~m} ; 1 \mathrm{H}), 3.16$ (br. s; $10-\mathrm{H}) .15 \mathrm{~b}: \delta=1.93$ (dt, $J_{3,4}=8.5, J_{2,3}=J_{3,5}=1.5 \mathrm{~Hz} ; 3-\mathrm{H}$ ), $2.13-2.21(\mathrm{~m} ; 1 \mathrm{H}), 2.21-2.28(\mathrm{~m} ; 1 \mathrm{H}), 2.32\left(\mathrm{dq}, J_{2,5}=4.0 \mathrm{~Hz}\right.$, Kopplungen zu 3-, 4-H und 1 oder $6-\mathrm{H}=1.5 \mathrm{~Hz} ; 2$ - oder $5-\mathrm{H}), 2.64\left(\mathrm{br} . \mathrm{dd}, J_{1,10}=6.7, J_{1,6}=7.5 \mathrm{~Hz} ; 1-\mathrm{H}\right), 2.78-2.83(\mathrm{~m}$; $1 \mathrm{H}), 3.30(\mathrm{~d}, 10-\mathrm{H}) .-{ }^{13} \mathrm{C}-\mathrm{NMR}\left(\mathrm{CDCl}_{3}\right.$, die Zuordnung von C-3 und $\mathrm{C}-4$ erfolgte in Analogie zu $14 \mathrm{a}, \mathrm{b}$ und $16 \mathrm{a}, \mathrm{b}): 15 \mathrm{a}: \delta=0.2(\mathrm{~d} ; \mathrm{C}-4), 9.5(\mathrm{~d} ; \mathrm{C}-3), 34.6$ und $35.0(\mathrm{~d} ; \mathrm{C}-2,-5), 45.9$ (d;C-1), 50.3 (d; C-6), 59.6 (d; C-10), 69.0 (s; C-7), 129.9 und 130.0 (jeweils s; C-8, -9). 15 b: $\delta=2.8$ (d; C-3), 4.0 (d; C-4), 34.1, 35.1 und 35.4 (jeweils d; C-1, -2, -5), 49.4 (d; C-6), 53.9 (d; C-10), 66.0 (s; C-7), 126.3 und 126.9 (jeweils s; $C-8,-9$ ).

$$
\mathrm{C}_{10} \mathrm{H}_{7} \mathrm{Cl}_{3} \text { (233.5) Ber. C } 51.43 \text { H } 3.02 \text { Gef. C } 51.95 \text { H } 3.30
$$

7,8,9,10-Tetrachlor-exo- (16a) und -endo-pentacyclo[4.4.0.0 $\left.0^{2,4} \cdot 0^{3.5} \cdot 0^{7,10}\right]$ dec-8-en (16 b): $1.53 \mathrm{~g}(5.71 \mathrm{mmol}) 13$ wurden bei $20^{\circ} \mathrm{C}$ in $250 \mathrm{ml}$ absol. Ether und $10 \mathrm{ml}$ Triethylamin nach Săttigen mit $\mathrm{N}_{2}$ durch Pyrex bis zur vollständigen Umwandlung (208 h) mit 320-nm-Licht bestrahlt (Gräntzel-Reaktor 400). Durch Extrahieren mit Wasser wurde eine Trübung entfernt. Nach dem Trocknen über $\mathrm{Na}_{2} \mathrm{CO}_{3}$ engte man im Rotationsverdampfer ein und unterwarf den Ruckstand der Destillation. Bei $70-110^{\circ} \mathrm{C}(\mathrm{Bad}) / 0.01$ Torr gingen $1.26 \mathrm{~g}(82 \%)$ einer farblosen, teilweise kristallisierenden Flüssigkeit über, deren NMR-Spektren 16a und 16b im Verhăltnis 40:60 identifizierten. Tieftemperaturkristallisation aus $n$-Pentan erbrachte farblose Kristalle mit Schmp. $39-50^{\circ} \mathrm{C}$. - IR (Film): 3130 (w), 3045 (w), 2955 (m) (jeweils C-H); $1605 \mathrm{~cm}^{-1}$ (s) (C=C). MS $(70 \mathrm{eV}): m / e=270,268,266\left(0.2 \%, 0.6 \%, 0.7 \%, M^{+}\right), 232(12), 230(12), 216(7), 200,198$, $196(12,68,100, \mathrm{M}-2 \mathrm{Cl}), 192(39), 190(81), 188$ (64), 160 (47), $126(35), 98$ (29), 74 (40), 61 (25), $52(61) .-{ }^{1} \mathrm{H}-\mathrm{NMR}\left(\mathrm{CDCl}_{3}\right): \delta=2.00-2.25(\mathrm{~m}), 2.25-2.50(\mathrm{~m}), 2.88$ (br. s; 1-, 6-H von 16b), $2.97(\mathrm{~m} ; 1-, 6-\mathrm{H}$ von $16 \mathrm{a}),-{ }^{13} \mathrm{C}-\mathrm{NMR}\left(\mathrm{CDCl}_{3}\right.$; die Unterscheidung der Signale von $\mathrm{C}-3$

Chem. Ber. 118 (1985) 
und $C-4$ beruht auf ihrer unterschiedlichen Feinstrukturad,1 bb): $16 \mathrm{a}: \delta=1.7(\mathrm{~d}, 221 \mathrm{~Hz} ; \mathrm{C}-4)$, 10.0 (d, $218 \mathrm{~Hz} ; \mathrm{C}-3), 33.7$ (d, $\approx 170 \mathrm{~Hz} ; \mathrm{C}-2,-5), 52.1$ (d, $149 \mathrm{~Hz} ; \mathrm{C}-1,-6), 75.0$ (s; C-7, -10), 132.0 (s; C-8, -9). 16b: $\delta=2.4$ (d, $221 \mathrm{~Hz} ; \mathrm{C}-4$ ), 5.9 (d, $218 \mathrm{~Hz} ; \mathrm{C}-3), 34.4$ (d, ₹ $170 \mathrm{~Hz} ; \mathrm{C}-2$, $-5), 48.7$ (d, $152 \mathrm{~Hz} ; \mathrm{C}-1,-6), 71.5$ (s; C-7, -10), 128.6 (s; C-8, -9).

$\mathrm{C}_{10} \mathrm{H}_{6} \mathrm{Cl}_{4}(268.0)$ Ber. C $44.82 \mathrm{H} 2.27 \mathrm{Cl} 52.92$ Gef. C $44.59 \mathrm{H} 2.35 \mathrm{Cl} 52.57$

Thermolyse des $14 \mathrm{a}, \mathrm{b}$-Gemisches: $2.7 \mathrm{mg} 14 \mathrm{a}, \mathrm{b}$ in $10 \mathrm{ml}$ Cyclohexan wurden in mehreren abgeschmolzenen Ampullen auf $120^{\circ} \mathrm{C}$ erhitzt. Den Fortgang der Reaktion verfolgte man anhand des charakteristischen UV-Spektrums ${ }^{4 a)}$ des entstandenen 7. 14a, b absorbieren bei Wellenlängen $>225 \mathrm{~nm}$ nicht nennenswert. Die Halbwertszeit der Reaktion betrug ca. $4 \mathrm{~h}$.

Thermolyse des 16a, b-Gemisches: a) $4.8 \mathrm{mg} \mathrm{16a,b}$ in $10 \mathrm{ml}$ Cyclohexan wurden in mehreren abgeschmolzenen Ampullen unter UV-Kontrolle (UV-Spektrum von 13 siehe Lit. 4 a); 16a, b absorbieren bei Welleniängen $>250 \mathrm{~nm}$ nicht nennenswert) auf $120^{\circ} \mathrm{C}$ erhitzt. Die Halbwertszeit der Reaktion betrug ca. $6 \mathrm{~h}$. b) Die praktisch gleiche Halbwertszeit wurde gefunden, als man eine konzentrierte Lösung von 16a, b in $\mathrm{C}_{6} \mathrm{D}_{6}$ im abgeschmolzenen NMR-Rohr auf $120^{\circ} \mathrm{C}$ erhitzte.

1,8,12,13-Tetrachlorhexacyclo[6.3.2.0 2,7.03,9. $\left.0^{4,6} \cdot 0^{9,11}\right]$ tridec-12-en (17): Nach Closs und Krant $z^{43)}$ wurde aus $43.0 \mathrm{~g}(562 \mathrm{mmol})$ Allylchlorid in $30 \mathrm{ml}$ Ligroin und $73.0 \mathrm{~g} \mathrm{30proz.} \mathrm{Natrium-}$ amid-Suspension innerhalb von $5 \mathrm{~h}$ Cyclopropen erzeugt und in eine auf $0^{\circ} \mathrm{C}$ gekühlte Lösung von $2.00 \mathrm{~g}(7.46 \mathrm{mmol}) 13 \mathrm{in} 80 \mathrm{ml}$ absol. Tetrahydrofuran geleitet. In Abänderung der Vorschrift wurde dic Natriumamid-Suspension auf $100^{\circ} \mathrm{C}$ (Bad) gehalten. Um entstandenes Ammoniak zu entfernen, wurde der mit Stickstoff verdünnte Gasstrom zuerst durch halbkonzentrierte Schwefelsäure und dann durch ein mit Phosphorpentoxid beschicktes Rohr geleitet. Nach dem Abdampfen des Tetrahydrofurans i. Vak. kristallisierte man den Rückstand aus Ethanol um, wobei $1.70 \mathrm{~g}(74 \%)$ farblose Kristalle von 17 mit Schmp. $132-134^{\circ} \mathrm{C}$ anfielen. - IR (KBr): $3142(\mathrm{w})$, $3065(\mathrm{w}), 3052(\mathrm{~m}), 3012(\mathrm{w}), 2933(\mathrm{~m})$ (jeweils $\mathrm{C}-\mathrm{H}) ; 1602 \mathrm{~cm}^{-1}$ (s) $(\mathrm{C}=\mathrm{C}),-$ MS (70 eV): $m / e=310,308,306\left(0.1 \%, 0.3 \%, 0.2 \%, \mathrm{M}^{+}\right), 277,275,273,271(0.7,7,21,23, \mathrm{M}-\mathrm{Cl}), 243$ (22), 237 (28), 236 (24), 235 (39), 202 (21), 201 (40), $200(54), 199(40), 197(31), 195(93), 193$ (100), $166(23), 165$ (75), $125(26), 82(21), 78$ (42), 77 (23), 63 (20), 52 (28), 51 (29), 39 (29). ${ }^{\mathrm{i}} \mathrm{H}-\mathrm{NMR}\left(\mathrm{CDCl}_{3}\right): \delta=0.69\left(\mathrm{q}, J_{10 \mathrm{a}, 10 \mathrm{~b}}=J_{9,10 \mathrm{~b}}=7.2 \mathrm{~Hz} ; 10 \mathrm{~b}-\mathrm{H}\right), 0.74\left(\mathrm{dt}, J_{9,10 \mathrm{a}}=4.3 \mathrm{~Hz}\right.$; 10a-H), $1.65(\mathrm{dd} ; 9-, 11-\mathrm{H}), 1.90\left(\mathrm{~m} ; J_{4,5} \approx 9 \mathrm{~Hz} ; 4\right.$ - oder 5-H), $2.12(\mathrm{~m} ; 3-, 6-\mathrm{H}$ und 5- oder 4-H), 2.68 (br. s; 2-, 7-H). $-{ }^{13} \mathrm{C}-\mathrm{NMR}$ ( $\mathrm{CDCl}_{3}$ ): $\delta=0.6$ (d, $216.2 \mathrm{~Hz} ; \mathrm{C}-4$ ), 3.5 (d, $219.1 \mathrm{~Hz}$; C-5, Zuordnung aufgrund der Feinstruktur $\left.{ }^{4 a, 11 b)}\right), 6.7$ (t, $\left.162.9 \mathrm{~Hz} ; \mathrm{C}-10\right), 23.4$ (d, $177.9 \mathrm{~Hz}$; C-9, -11), 34.1 (d, 168.4 Hz; C-3, -6), 57.9 (d, $139.6 \mathrm{~Hz} ; \mathrm{C}-2,-7), 71.8$ (s; C-1, -8), 123.6 (s; C-12, $-13)$.

$$
\mathrm{C}_{13} \mathrm{H}_{10} \mathrm{Cl}_{4} \text { (308.1) Ber. C } 50.69 \text { H } 3.27 \text { Gef. C } 50.85 \text { H } 3.33
$$

$1,8,14,15$-Tetrachlor-11-methyl-9, 11,13-triazahexacyclo[6.5.2. $\left.0^{2,7} \cdot 0^{3,5} \cdot 0^{4,6} \cdot 0^{9,13}\right]$ pentadec-14en-10,12-dion (18): Unter Stickstoff und Rühren wurde bei Raumtemp. die Lösung von $1.50 \mathrm{~g}$ $(5.60 \mathrm{mmol}) 13$ in $80 \mathrm{ml}$ absol. Dichlormethan portionsweise mit $630 \mathrm{mg}(5.60 \mathrm{mmol}) 4$-Methyl$4 H$-triazol-3,5-dion ${ }^{44)}$ versetzt. Dabei wartete man mit der neuen Zugabe jeweils, bis die Färbung des Dienophils abgebaut war. Am Ende blieb ein blasses Rot bestehen. Man engte im Rotationsverdampfer ein und kristallisierte aus Methanol um, was zu $1.85 \mathrm{~g}(87 \%)$ farblosem $18 \mathrm{mit}$ Schmp. $205-207^{\circ} \mathrm{C}$ führte. - IR (KBr): 3150 (w), $3120(\mathrm{~m}), 3060(\mathrm{w}), 3045(\mathrm{w}), 2963(\mathrm{w}), 2942$ (w) (jeweils C-H); 1795 (s), 1730 (s) (jeweils $C=0) ; 1685 \mathrm{~cm}^{-1}(\mathrm{~m})(\mathrm{C}=\mathrm{C}) .-\mathrm{MS}(70 \mathrm{eV})$ : $m / e=383,381\left(0.1 \%, 0.2 \%, M^{+}\right), 233(4), 231(4), 218(4), 198(6), 196(9), 186(7), 160(4), 126$ (3), $87(4), 53(5), 52\left(100, \mathrm{C}_{4} \mathrm{H}_{4}\right), 51(8), 50(4), 39(4) .-{ }^{1} \mathrm{H}-\mathrm{NMR}\left(\mathrm{CDCl}_{3}\right): \delta=2.20$ und 2.30 (jeweils m; jeweils $2 \mathrm{H}, 3-, 6-\mathrm{H}, 4-\mathrm{S}-\mathrm{H}), 2.86$ (br. s; 2-, 7-H), 3.01 (s; $\left.\mathrm{CH}_{3}\right) .-{ }^{13} \mathrm{C}-\mathrm{NMR}$ $\left(\mathrm{CDCl}_{3}\right): \delta=0.1$ und $2.4(\mathrm{C}-4,-5), 26.1\left(\mathrm{CH}_{3}\right), 34.4(\mathrm{C}-3,-6), 53.1(\mathrm{C}-2,-7), 83.0(\mathrm{C}-1,-8), 126.8$ $(\mathrm{C}-14,-15), 154.2(\mathrm{C}=0)$.

$\mathrm{C}_{13} \mathrm{H}_{9} \mathrm{Cl}_{4} \mathrm{~N}_{3} \mathrm{O}_{2}(381.1)$ Ber. C 40.97 H $2.38 \mathrm{~N} 11.03$ Gef. C 40.91 H $2.38 \mathrm{~N} 10.89$ 


\section{Darstellung der Benzobenzvalene ${ }^{45)}$ und des Diketons 32}

8-Chlortetracyclo/4.4.0.0 $0^{2,4}, 0^{3,5}$ Jdeca-1(6), 7,9-trien (19): Zur Lösung von $2.50 \mathrm{~g}(12.6 \mathrm{mmol})$ 11 in $35 \mathrm{ml}$ absol. Dimethylsulfoxid (DMSO) wurden unter Rühren innerhalb von $10 \mathrm{~min} 3.00 \mathrm{~g}$ (26.7 mmol) Kalium-tert-butoxid in $35 \mathrm{ml}$ absol. DMSO getropft, wobei Braunschwarzfärbung und Erwärmung auf ca. $50^{\circ} \mathrm{C}$ eintraten. Nach weiteren 30 min Rühren wurde das Gemisch auf Eis gegossen und nach dessen Schmelzen $3 \mathrm{mal}$ mit Cyclohexan extrahiert. Einen schleimigen braunen Niederschlag entfernte man aus den vereinigten Cyclohexanphasen durch Filtration und schüttelte $5 \mathrm{mal}$ mit Wasser aus. Nach dem Trocknen über $\mathrm{CaCl}_{2}$ wurde die Cyclohexanlösung durch basisches $\mathrm{Al}_{2} \mathrm{O}_{3}$ (Akt.-Stufe III) filtriert und i. Vak. eingeengt. Der gelbe flüssige Ruckstand lieferte durch Destillation bei $60-70^{\circ} \mathrm{C}$ (Bad) $/ 0.1$ Torr $1.40 \mathrm{~g}(68 \%) 19$ als farblose Flüssigkeit, die bei Kuhlung zu Kristallen mit Schmp. $20-22^{\circ} \mathrm{C}$ erstarrte. - IR $\left(\mathrm{CDCl}_{3}\right): 3078(\mathrm{~m})$, $2925(\mathrm{w})$ (jeweils $\mathrm{C}-\mathrm{H}) ; 1615(\mathrm{~m}), 1590 \mathrm{~cm}^{-1}(\mathrm{~m})$ (jeweils $\left.\mathrm{C}=\mathrm{C}\right) .-\mathrm{MS}(70 \mathrm{eV}): \mathrm{m} / \mathrm{e}=164,162$ $\left(33 \%, 100 \%, \mathrm{M}^{+}\right), 127(46), 126(19), 81(9), 63(11) .-{ }^{1} \mathrm{H}-\mathrm{NMR}$ : Tab. 1; Banden von Verunreinigungen in geringer Menge finden sich bei $\delta=7.2-7.7$ und 2.7, die möglicherweise auf Chlornaphthalin bzw. das 7-Chlorisomere von 19 zuruickgehen. $-{ }^{13} \mathrm{C}$-NMR: Tab. 2.

$$
\mathrm{C}_{10} \mathrm{H}_{7} \mathrm{Cl}(162.6) \text { Ber. C } 73.86 \mathrm{H} 4.34 \text { Gef. C } 73.42 \mathrm{H} 4.41
$$

8,9-Dichlortetracyclo[4.4.0.0 $0^{2,4} \cdot 0^{3,5}$ Jdeca-1(6),7,9-trien (20): Wie bei 19 beschrieben, wurden $1.30 \mathrm{~g}(5.56 \mathrm{mmol}) 12$ in $10 \mathrm{ml}$ absol. DMSO mit $1.50 \mathrm{~g}(13.4 \mathrm{mmol})$ Kalium-tert-butoxid in $20 \mathrm{ml}$ absol. DMSO umgesetzt. Die analoge Aufarbeitung erbrachte nach Destillation bei $50-60^{\circ} \mathrm{C}$ (Bad) $/ 0.001$ Torr $470 \mathrm{mg}(43 \%) 20$ als farblose Flüssigkeit, die zu Kristallen mit Schmp. $65-66^{\circ} \mathrm{C}$ erstarrte. - MS $(70 \mathrm{eV}): m / e=200,198,196\left(11 \%, 65 \%, 100 \%, \mathrm{M}^{+}\right), 163(17), 161$ (50), 160 (14), 126 (55), 125 (15), 99 (14), 98 (13). - ${ }^{1}$ H-NMR: Tab. 1. - ${ }^{13}$ C-NMR: Tab. 2.

$$
\mathrm{C}_{10} \mathrm{H}_{6} \mathrm{Cl}_{2} \text { (197.1) Ber. C } 60.95 \mathrm{H} 3.07 \text { Gef. C } 60.71 \mathrm{H} 3.33
$$

7,8,9-Trichlor- (21) und 7,8,10-Trichlortetracyclo/4.4.0.0.0 $2,4.0^{3,5}$ Jdeca-1(6), 7,9-trien (22): Wie bei 19 beschrieben, wurden $3.00 \mathrm{~g}(11.2 \mathrm{mmol}) 13$ in $60 \mathrm{ml}$ absol. DMSO mit $3.00 \mathrm{~g}(26.7 \mathrm{mmol})$ Kalium-tert-butoxid in $40 \mathrm{ml}$ absol. DMSO umgesetzt. Die analoge Aufarbeitung lieferte nach Destillation bei $70-80^{\circ} \mathrm{C}(\mathrm{Bad}) / 0.01$ Torr $1.40 \mathrm{~g}(54 \%)$ einer farblosen Flüssigkeit, die bei Kühlung zur Kristallen mit Schmelzintervall $43-50^{\circ} \mathrm{C}$ erstarrte. Die NMR-Analyse zeigte 21 und 22 im Verhältnis 5:1 an. - IR (Film): $3110(w), 3075(w)$, (jeweils C - H); $1610(w), 1565 \mathrm{~cm}^{-1}$ (br., m) (jeweils $C=C)$. - MS $(70 \mathrm{eV}): m / e=236,234,232,230\left(3 \%, 31 \%, 95 \%, 100 \%, \mathrm{M}^{+}\right), 197$ (20), $196(16), 195(30), 162(15), 160$ (47), 125 (13), 98 (12), 80 (11). - ${ }^{1}$ H-NMR: Tab. $1 .-{ }^{13} \mathrm{C}-$ NMR: Tab. 2.

$$
\mathrm{C}_{10} \mathrm{H}_{5} \mathrm{Cl}_{3} \text { (231.5) Ber. C } 51.88 \mathrm{H} 2.18 \text { Gef. C } 51.96 \mathrm{H} 2.23
$$

$\left[3,4,10-D_{3}\right]-7,8,9-$ Trichlor- (21 a) und $\left[3,4,9-D_{3}\right]-7,8,10$-Trichlorletracyclo[4.4.0.0.0.4. $\left.0^{3,5}\right]$ deca-1(6), 7,9-trien (22a): Wie bei der Synthese von 21 und 22 wurden aus $300 \mathrm{mg}(1.12 \mathrm{mmol}) 13$ und $300 \mathrm{mg}$ Kalium-tert-butoxid in insgesamt $6 \mathrm{ml}$ [D]DMSO $150 \mathrm{mg}(58 \%)$ 21 a und 22a erhalten. Das ${ }^{1} \mathrm{H}$-NMR-Sektrum zeigt ein intensives AB-System mit $J=5.5 \mathrm{~Hz}$ bei $\delta=2.58$ und 2.81 (21 a) sowie ein wenig intensives AB-System mit $J=5.7 \mathrm{~Hz}$ bei $\delta=2.81$ und 2.84 (22a).

8,9-Dichlor-10-hydroxytetracyclo[4.4.0.0 $0^{2,4} \cdot 0^{3,5}$ Jdeca-1(6),7,9-trien-7-carbonsäure-methylester (27): Unter Stickstoff und Rühren wurden zu $2.76 \mathrm{~g}$ (51.1 mmol) Natriummethoxid in $15 \mathrm{ml}$ absol. Methanol bei $-3^{\circ} \mathrm{C}$ innerhalb von $2 \mathrm{~h} 2.00 \mathrm{~g}(6.41 \mathrm{mmol}) 10 \mathrm{in} 30 \mathrm{ml}$ absol. Ether getropft. Unter Rühren ließ man auf $20^{\circ} \mathrm{C}$ kommen (30 min), versetzte mit $20 \mathrm{ml}$ Wasser und gab bis zum Erreichen des Neutralpunkts Essigsäure zu. Extraktion $(2 \times 30 \mathrm{ml})$ mit Ether, Trocknen der Etherphase mit $\mathrm{Na}_{2} \mathrm{SO}_{4}$ und Einengen $\mathrm{i}$. Vak. lieferten braune Kristalle. Mehrmaliges Umkristallisieren aus Ethanol erbrachte $940 \mathrm{mg}(54 \%) 27$ in Form farbloser Kristalle mit Schmp. $137-139^{\circ} \mathrm{C}$. - IR (KBr): 3400 (br., m, O- H); 3107, 3095, 3000, 2952, 2845 (jeweils w, C - H); $1700(\mathrm{~s}, \mathrm{C}=\mathrm{O}) ; 1600,1580 \mathrm{~cm}^{-1}$ (jeweils $\left.\mathrm{m}, \mathrm{C}=\mathrm{C}\right) .-\mathrm{MS}(70 \mathrm{eV}): \mathrm{m} / \mathrm{e}=274,272,270(3 \%$, 
$\left.16 \%, 25 \%, M^{+}\right), 243,241,239\left(11,66,100, M-O C H_{3}\right), 211(11), 183(15), 175(17), 149(12)$, 148 (11), 147 (24), 113 (16). - ${ }^{1}$ H-NMR: Tab. 1. - ${ }^{13}$ C-NMR: Tab. 2.

$\mathrm{C}_{12} \mathrm{H}_{8} \mathrm{Cl}_{2} \mathrm{O}_{3}$ (271.1) Ber. C $53.16 \mathrm{H} 2.97$ Gef. C $53.50 \mathrm{H} 3.28$

\section{7,10-Diphenyl-8,9-diazatetracyclo[4.4.0.0 2,4:03,5]deca-1(6),7,9-trien (29) aus 284a)}

a) Mit DDQ: Unter Luft- und Feuchtigkeitsausschluß versetzte man $1.00 \mathrm{~g}(3.52 \mathrm{mmol}) 28$ in $30 \mathrm{ml}$ absol. Benzol mit $870 \mathrm{mg}$ (3.83 mmol) DDQ in $40 \mathrm{ml}$ absol. Benzol, wobei sofort Erwărmung und Abscheidung einer Teerschicht eintraten. Nach Rückflußkochen über Nacht filtrierte man, extrahierte das Filtrat mit Natriumhydrogensulfit-Lösung und dampfte das Benzol i. Vak. ab. Es blieb ein rotbrauner Feststoff zurück, dessen Umkristallisation aus Ethanol $420 \mathrm{mg} \mathrm{(42 \% )}$ 29 lieferte. Nach Chromatographie an Kieselgel mit Dichlormethan/Ethylacetat (10:1) schmolzen die gelblichen Prismen bei $207-210^{\circ} \mathrm{C}$, wobei ab $185^{\circ} \mathrm{C}$ Braunfärbung eintrat. - IR (KBr): $3065(w), 1578(w), 1560(\mathrm{~m}), 1493(\mathrm{~m}), 1444(w), 1371(\mathrm{~s}), 1315(w), 1165(w), 1098(w), 1083(\mathrm{~m})$, $1069(\mathrm{w}), 1037(\mathrm{~m}), 896(\mathrm{~m}), 779(\mathrm{~m}), 769(\mathrm{~m}), 749(\mathrm{~m}), 729(\mathrm{~m}), 719(\mathrm{~m}), 708(\mathrm{~m}), 696(\mathrm{~s})$, $635 \mathrm{~cm}^{-1}(\mathrm{w}) .-\mathrm{UV}\left(\mathrm{CH}_{3} \mathrm{CN}\right): \lambda_{\max }(\lg \varepsilon)=253 \mathrm{~nm}(4.09)$. $-\mathrm{MS}(70 \mathrm{eV}): m / e=282(60 \%$, $\mathrm{M}^{+}$), 281 (100), 252 (17), 126 (13), 77 (14). - ${ }^{1}$ H-NMR: Tab. 1. - ${ }^{13}$ C-NMR: Tab. 2.

$$
\mathrm{C}_{20} \mathrm{H}_{14} \mathrm{~N}_{2} \text { (282.3) Ber. C } 85.08 \text { H } 5.00 \mathrm{~N} 9.92 \text { Gef. C } 84.99 \text { H } 4.81 \text { N } 9.63
$$

b) Mit Fenchon/Kaliumfencholat: Man vereinigte $40 \mathrm{mg}$ (1.00 mmol) Kaliumhydrid und $445 \mathrm{mg}$ (2.99 mmol) Fenchon in $15 \mathrm{ml}$ absol. DMSO und rührte $30 \mathrm{~min}$. Dann tropfte man langsam $700 \mathrm{mg}(2.46 \mathrm{mmol}) 28 \mathrm{in} 30 \mathrm{ml}$ absol. DMSO zu und erhitzte $23 \mathrm{~h}$ auf $70^{\circ} \mathrm{C}$. Zur Aufarbeitung gab man Wasser zu, extrahierte $4 \mathrm{mal}$ mit Cyclohexan, extrahierte die vereinigten Cyclohexanphasen $3 \mathrm{mal}$ mit Wasser und trocknete mit $\mathrm{Na}_{2} \mathrm{SO}_{4}$. Nach dem Einengen i. Vak. blieb ein braunes $\mathrm{Ol}$ zurück, aus dem bis $50^{\circ} \mathrm{C}(\mathrm{Bad}) / 0.001$ Torr flüchtige Verbindungen (Fenchol) abdestilliert wurden. Der Rückstand ( $250 \mathrm{mg}, 36 \%$ ) erwies sich als 29, identisch mit dem Produkt aus a).

c) Mit Dehydrobenzol: $12.0 \mathrm{mmol}$ 1-Benzoldiazonium-2-carboxylat ${ }^{46)}$ wurden mit $1.70 \mathrm{~g}$ $(5.99 \mathrm{mmol}) 28$ in $50 \mathrm{ml}$ absol. Dichlormethan vereinigt, wobei eine spontane Gasentwicklung eintrat. Man hielt die Lơsung $4 \mathrm{~h}$ auf $40^{\circ} \mathrm{C}$, ließ über Nacht bei Raumtemp. stehen und entfernte das Lösungsmittel i. Vak. Der rotbraune Rückstand enthielt neben Biphenylen ( ${ }^{1} \mathrm{H}-\mathrm{NMR}$ in $\mathrm{CDCl}_{3}: \delta=6.60$ und 6.70) nur 29. Säulenchromatographie über Kieselgel mit Dichlormethan/ Essigester (5:1) gestattete die Trennung, und nach Umlosen aus Ethanol fielen $750 \mathrm{mg}(44 \%) 29$. identisch mit dem Produkt aus a), an.

d) Durch Belichten: $1.50 \mathrm{~g}(5.28 \mathrm{mmol}) 28$ in $300 \mathrm{ml}$ absol. Benzol wurden nach Spülen mit Stickstoff bei $20^{\circ} \mathrm{C} 35 \mathrm{~h}$ durch Pyrex belichtet (Hanovia $450 \mathrm{~W}$ Hg-Mitteldrucklampe). Das Einengen i. Vak. hinterließ $820 \mathrm{mg}$ Rohprodukt, aus dem durch Filtration der Benzollösung uber $\mathrm{Al}_{2} \mathrm{O}_{3}$ (Akt.-Stufe III) und Umlosen reines 29 gewonnen wurde.

cis-3,4-Dibenzoyltricyclo/3.1.0.0 2,6 Jhexan (32): $\mathrm{Zu} 1.50 \mathrm{~g}$ (8.71 mmol) 80proz. m-Chlorperbenzoesäure in $20 \mathrm{ml}$ absol. Dichlormethan tropfte man bei $0^{\circ} \mathrm{C}$ unter Rühren innerhalb von $30 \mathrm{~min}$ die gelbe Losung von $1.00 \mathrm{~g}(3.52 \mathrm{mmol}) 28 \mathrm{in} 10 \mathrm{ml}$ absol. Dichlormethan, wobei sofort Entfärbung eintrat und ein farbloser Niederschlag ausfiel. Nach $24 \mathrm{~h}$ Rühren bei $20^{\circ} \mathrm{C}$ wurde filtriert, die Lơsung im Rotationsverdampfer eingeengt und der Ruckstand an Kieselgel mit Dichlormethan/Ethylacetat (10:1) chromatographiert. Nach Umlosen aus Ethanol erhielt man $310 \mathrm{mg}$ $(30 \%) 32$ als farblose verfilzte Nadeln mit Schmp. $164-166^{\circ} \mathrm{C}$. - IR (KBr): $1680 \mathrm{~cm}^{-1}$ $(C=O)$. $-M S(70 \mathrm{eV}): m / e=288\left(5 \%, \mathrm{M}^{+}\right), 183(22), 105\left(100, \mathrm{C}_{6} \mathrm{H}_{5}-\mathrm{CO}\right), 77(51), 51(10)$. ${ }^{1} \mathrm{H}-\mathrm{NMR}\left(\mathrm{CDCl}_{3}\right): \delta=2.13$ und 2.45 (jeweils br. d; $J_{1,6}=9.0 \mathrm{~Hz}$; 1 - und 6-H), 2.53 (br. s; 2-, 5-H), 3.93 (br. s; 3-, 4-H), $7.20-7.44$ (m; 6 aromat. H), $7.68-7.94$ (m; 4 aromat. H). $-{ }^{13} \mathrm{C}-$ 
NMR $\left(\mathrm{CDCl}_{3}\right): \delta=2.9$ und 4.9 (jeweils d; $C-1$ und -6 ), 38.5 (d; C-2, -5), 52.9 (d; C-3, -4), 127.8 und 128.4 (jewejls $d ; C-2^{\prime}$ und $-3^{\prime}$ ), 132.4 (d; C-4'), 137.9 (s; C-1'), 199.0 (s; $C=O$ ).

$$
\mathrm{C}_{20} \mathrm{H}_{16} \mathrm{O}_{2} \text { (288.3) Ber. C } 83.31 \text { H } 5.59 \text { Gef. C } 83.07 \text { H } 5.41
$$

Reaktion von 28 mit Sauerstoff: Durch eine Lösung von $500 \mathrm{mg}(1.76 \mathrm{mmol}) 28 \mathrm{in} 100 \mathrm{ml}$ absol. Benzol ließ man bei $20^{\circ} \mathrm{C} 7.5 \mathrm{~h}$ trockenen Sauerstoff perlen, wobei die ursprünglich gelbe Losung verblaßte. Nach dem Einengen zeigte das ${ }^{1}$ H-NMR-Spektrum des Rückstandes den vollständigen Umsatz von 28 an. Aufgrund der quantitativen NMR-Analyse mit Fumarsäure-dimethylester als internem Standard enthielt das Rohprodukt $164 \mathrm{mg}$ (32\%) 32 und $79 \mathrm{mg}$ (17\%) 29. Durch Chromatographie an Kieselgel mit Dichlormethan/Ethylacetat $(5: 1)$ wurde das Gemisch getrennt. 32 hat die kürzere, 29 die längere Retentionszeit.

In Dichlormethan und Acetonitril verlief die Reaktion analog. Durch einen Zusatz an festem Kaliumcarbonat (Rühren!) wurde sie in allen drei Lösungsmitteln stark verlangsamt.

Beim Rühren von $50 \mathrm{mg} 28$ in $3 \mathrm{ml}$ Dichlormethan bei $20^{\circ} \mathrm{C}$ im offenen Kolben über $24 \mathrm{~h}$ wurde 28 vollständig verbraucht, und es bildeten sich 32 und 29 im Verhältnis $2: 1$. Die Wiederholung dieses Versuchs mit $100 \mathrm{mg} 28$ in $10 \mathrm{ml}$ Dichlormethan erbrachte 32 und 29 im Verhältnis $1: 1$ und ein drittes Produkt, das im ${ }^{1}$ H-NMR-Spektrum Banden bei $\delta=0-2$ sowie je ein enges Multiplett bei $\delta=4.95$ und 6.78 aufwies. Es handelte sich vermutlich um 3,4-Dibenzoylbicyclo[3.1.0]hex2-en, dessen Bildung aus 32 unter Säurekatalyse verständlich wäre.

$$
\begin{aligned}
& \text { 8,9-Diazatetracyclo[4.4.0.02,4 } .0^{3,5} \text { ]deca-1(6),7,9-trien-7,10-dicarbonsäure-dimethylester } \\
& \text { aus 304a) }
\end{aligned}
$$

a) Mit DDQ: Unter Stickstoff wurden $2.00 \mathrm{~g}(8.06 \mathrm{mmol}) 30$ und $2.00 \mathrm{~g}(8.81 \mathrm{mmol}) \mathrm{DDQ}$ in $200 \mathrm{ml}$ absol. Benzol $16 \mathrm{~h}$ am Rückflußkühler gekocht. Man filtrierte den Niederschlag ab, extrahierte das Filtrat zweimal mit je $125 \mathrm{ml}$ wäßriger Natriumhydrogensulfit-Lơsung und dann einmal mit Wasser. Nach dem Trocknen mit $\mathrm{Na}_{2} \mathrm{SO}_{4}$ hinterließ das Verdampfen des Lösungsmittels i. Vak. $1.43 \mathrm{~g}$ braune Kristalle. Umlösen aus viel Aceton lieferte $1.20 \mathrm{~g}(60 \%) 31 \mathrm{in}$ Form rótlicher Kristalle mit Schmp. $185-187^{\circ} \mathrm{C}$. - IR (KBr): $1746(\mathrm{~m}), 1720(\mathrm{~s}), 1708 \mathrm{~cm}^{-1}$ (s) (jeweils $\mathrm{C}=\mathrm{O}) .-\mathrm{UV}\left(\mathrm{CH}_{3} \mathrm{CN}\right): \lambda_{\max }(\mathrm{lg} \varepsilon)=312(2.75, \mathrm{sh}), 281 \mathrm{~nm}(3.32) .-\mathrm{MS}(70 \mathrm{eV}): m / e=246$ $\left(5 \%, \mathrm{M}^{+}\right), 188(9), 129(100), 102(10), 59(9) .-{ }^{1} \mathrm{H}-\mathrm{NMR}:$ Tab. 1. $-{ }^{13} \mathrm{C}-\mathrm{NMR}:$ Tab. 2.

$$
\mathrm{C}_{12} \mathrm{H}_{10} \mathrm{~N}_{2} \mathrm{O}_{4} \text { (246.2) Ber. C } 58.54 \mathrm{H} 4.09 \mathrm{~N} 11.38 \text { Gef. C } 58.47 \mathrm{H} 3.90 \mathrm{~N} 10.97
$$

b) Mit m-Chlorperbenzoesäure: Bei $0^{\circ} \mathrm{C}$ wurden $1.50 \mathrm{~g}(6.04 \mathrm{mmol}) 30$ in $40 \mathrm{ml}$ absol. Dichlormethan unter Rüren innerhalb von $2 \mathrm{~h}$ zu $2.60 \mathrm{~g}(12.1 \mathrm{mmol}) 80 \mathrm{proz}$. $m$-Chlorperbenzoesäure in $40 \mathrm{ml}$ absol. Dichlormethan getropft. Dabei wurde die gelbe 30-Lösung sofort entfärbt und ein farbloser Niederschlag fiel aus. Nach dem Aufwärmen auf $20^{\circ} \mathrm{C}$ beließ man $6 \mathrm{~h}$ bei dieser Temperatur. Nun wurde filtriert und die Lösung i. Vak. eingeengt. Chromatographie des Rückstandes an Kieselgel mit Ethylacetat erbrachte $500 \mathrm{mg} \mathrm{(34 \% )} 31$ als rơtliche Kristalle, die in allen Eigenschaften mit dem Produkt aus a) ubereinstimmten.

\section{Messung der ${ }^{13} \mathbf{C}$-NMR-Spektren}

Zur Ermittlung der chemischen Verschiebungen wurden die Protonen breitbandentkoppelt. Die ${ }^{13} \mathrm{C}$-H-Kopplungskonstanten bestimmte man mit Hilfe des "gated-decoupling"-Verfahrens (Bruker WH 90) oder ganz ohne Entkopplung (Bruker WM 400). Die Verbindungen 619, $35^{47}$ ) und 3948) stellten wir nach Literaturvorschriften her und nahmen ihre Spektren in $\mathrm{CDCl}_{3}$ auf. Bei 35 sicherten wir durch selektive Entkopplung von jeweils einem der beiden Bicyclo[1.1.0]butanH-Signale bei der Aufnahme des ${ }^{13} \mathrm{C}$-NMR-Spektrums deren Zuordnung. Bei den Signalen im aromatischen Bereich gingen wir davon aus, daß aufgrund der Erfahrungen beim Naphthalin 26b) von den weitreichenden Kopplungen nur die über drei, nicht aber die uiber zwei und vier oder mehr Bindungen aufgelöst sein sollten. Somit ergibt sich eine Reihenfolge der chemischen Ver-

Chem. Ber. 118 (1985) 
schiebungen, die der von 1,2-Dihydrophenalen ${ }^{49)}$ ähnlich ist, aber von jener des 1,8-Dimethylnaphthalins ${ }^{50)}$ doch beachtlich abweicht.

1) Aus der Diplomarbeit $S$. Brengel und der Dissertation S. Freund, Univ. Würzburg 1980 bzw. 1983.

2) $M$. Christl und R. Herbert, Org. Magn. Reson. 12, 150 (1979).

3) K. Tori, T. Tsushima, H. Tanida, K. Kushida und S. Satoh, Org. Magn. Reson. 6, 324 (1974).

4) 4a) $M$. Christl, H.-J. Lüddeke, A. Nagyrevi-Neppel und G. Freitag, Chem. Ber. 110, 3745 (1977). - 4b) Zusammenfassung aber Benzvalen: M. Christl, Angew. Chem. 93, 515 (1981); Angew. Chem., Int. Ed. Engl. 20, 529 (1981).

5) $M$. Christl und $R$. Herbert, Chem. Ber. 112, 2022 (1979).

6) $O$. Diels und $K$. Alder, Liebigs Ann. Chem. 490, 257 (1931). Weitere [4+2]-Addukte von a-Pyronen: $\boldsymbol{H}$. Wollweber, in Methoden der organischen Chemie (Houben-Weyl-Müller), 4. Aufl., Bd. V/1c, S. 977, Thieme, Stuttgart 1970; H. won Brachel und $U$. Bahr, cbenda S. 1 und zit. Lit.

7) J. Sauer und R. Sustmann, Angew. Chem. 92, 773 (1980); Angew. Chem., Int. Ed. Engl. 19. $779(1980)$.

8) G. Märkl, Chem. Ber. 96, 1441 (1963).

9) Hooker Chemical Corp. (Erf. E. Leon), US-Pat. 3092641 [Chem. Abstr. 60, 2902 b (1964)] und 3274131 [Chem. Abstr. 66, 65190u (1967)].

10) $H$. Schuster und J. Sauer, Tetrahedron Lett. 24, 4087 (1983).

11) 1ta) Analog zur Reaktionsfolge $4+$ Tetrachlor- $\alpha$-pyron $\rightarrow 10 \rightarrow 27$ wurden auch Cyclopentadien, Cyclopenten, Norbornen, Styrol und Ethylvinylether umgewandelt: $M$. Keilwerth, $A$. Conradi, Diplomarbeiten, Univ. Würzburg 1984. - 11b) M. Christl, $H$. Leininger und $P$. Kemmer, Chem. Ber. 117, 2963 (1984).

12) Dr. M. Kapon und Prof. M. Rubin, Technion - Israel Institute of Technology, Haifa, Privatmitteilung.

13) M. S. Raasch, J. Org. Chem. 45, 856 (1980).

14) H. Meier, in Methoden der organischen Chemie (Houben-Weyl-Müller), 4. Auf1., Bd. IV/5a, Teil I, S. 222, Thieme, Stuttgart 1975.

15) Die ersten Versuche zur Photolyse von 7 und 13 wurden 1976 freundlicherweise von Prof. G. Maier und Dr. H. P. Reisenauer, damals Univ. Marburg, jetzt Univ. Gießen, bei tiefer Temperarur in einer Matrix vorgenommen. Die IR-Spektren ließen die Bildung von 14 bzw. 16 vermuten.

16) T. J. Katz, E. J. Wang und N. Acton, J. Am. Chem. Soc. 93, 3782 (1971).

17) G. Gandillon, B. Bianco und U. Burger, Tetrahedron Lett. 22, 51 (1981).

18) U. Burger und F. Dreier, Helv. Chim. Acta 62, 540 (1979); Tetrahedron 39, 2065 (1983).

19) $U$. Burger und B. Bianco, Helv. Chim. Acta 66, 60 (1983).

20) 20a) Z. Yoshida, S. Miki, F. Kawamoto, T. Hijiya und H. Ogoshi, Koen Yoshishu - Hibenzenkei Hokozuku Kagaku Toronkai [oyobi] Kozo Yuki Kagaku Toronkai, $12^{\text {th }} 1979,313$ (Shinshu Daigaku Rigakubu: Matsumoto, Japan) [Chem. Abstr. 92, 163323 w (1980)]; Sekisiu Chemical Co., Ltd (Erf. Z. Yoshida, F. Kawamoto, H. Miyoshi und H. Ikikoshi), Jpn. Kokai Tokkyo Koho 79138549 [Chem. Abstr. 92, 163763 q (1980)]; Z. Yoshida, S. Miki, F. Kawamolo, T. Hijiya und H. Ogoshi, Kokagaku Toronkai Koen Yoshishu 1979, 6 (Chem. Soc. Japan: Tokyo, Japan) [Chem. Abstr. 92, 197660u (1980)]. - 20b) Z. Yoshida, Vortrag am 20.7.1982 in Würzburg.

21) W. P. Lay, K. Mackenzie und J. R. Telford, J. Chem. Soc. C 1971, 3199.

22) S. Sternhell, Q. Rev., Chem. Soc. 23, 236 (1969).

23) M. T. Reetz und F. Eibach, Liebigs Ann. Chem. 1978, 1598.

24) 24a) $P$. Lechtken, $Z$. Naturforsch., Teil B 31, $1436(1976) ; M$. E. Landis und D. C. Madoux, J. Am. Chem. Soc. 101, 5106 (1979); W. Ando, R. Sato, H. Sonobe und T. Akasaka, Tetrahedron Lett. 25, 853 (1984). - 24b) S. Satish, A. Mitra und M. V. George, Tetrahedron 35, 277 (1979); J. Baker, W. Hedges, J. W. Timberlake und L. M. Trefonas, J. Heterocycl. Chem. 20, 855 (1983), und zit. Lit.

25) L. Horner, $W$. Kirmse und $H$. Fernekess, Chem. Ber. 94, 279 (1961).

26) 26a) H. Günther, H. Seel und M. E. Günther, Org. Magn. Reson. 11, 97 (1978). - 26b) H. Seel, $R$. Aydin und $H$. Gïnther, Z. Naturforsch., Teil B 33, 353 (1978).

27) G. C. Lev'y, R. L. Lichter und G. L. Nelson, Carbon-13 Nuclear Magnetic Resonance Spectroscopy, Second Edition, John Wiley \& Sons, New York, Chichester, Brisbane, Toronto 1980.

Chem. Ber. 118 (1985) 
28) E. Heilbronner und $H$. Bock, Das HMO-Modell und seine Anwendung. Tabellen berechneter und experimenteller Großen, Verlag Chemie, Weinheim 1970.

29) 29a) $P$. Bischof, $R$. Gleiter und $E$. Müller, Tetrahedron 32, 2769 (1976). - 29b) $R$. Gleiter, K. Gubernator, M. Eckert-Maksic, J. Spanget-Larsen, B. Bianco, G. Gandillon und U. Burger, Helv. Chim. Acta 64, 1312 (1981).

30) M. N. Paddon-Row, K. N. Houk, P. Dowd, P. Garner und R. Schappert, Tetrahedron Lett. 22, 4799 (1981); P. G. Gassman, M. L. Greenlee, D. A. Dixon, S. Richtsmeier und J. Z. Gougoulas, J. Am. Chem. Soc. 105, 5865 (1983).

31) $H$. Babsch, $H$. Fritz und $H$. Prinzbach, Tetrahedron Lett. 1975, 4677.

32) Y. Sugihara, N. Morokoshi und I. Murata, Tetrahedron Lett. 1977, 3887.

33) $H$. Volz, J.-H. Shin, H. Prinzbach, H. Babsch und M. Christl, Tetrahedron Lett. 1978, 1247.

34) $M$. Christl, Chem. Ber. 108, 2781 (1975)

35) 35a) $M$. Christl und R. Lang, J. Am. Chem. Soc. 104, 4494 (1982). - 35b) F. Lanzendörfer und M. Christl, Angew. Chem. 95, 896 (1983); Angew. Chem., Int. Ed. Engl. 22, 871 (1983).

36) G. Maier, M. Hoppe und H. P. Reisenauer, Angew. Chem. 95, 1009 (1983); Angew. Chem., Int. Ed. Engl. 22, 990 (1983).

37) K. Wüthrich, S. Meiboom und L. C. Snyder, J. Chem. Phys. 52, 230 (1970).

38) $M$. Christl, H. Leininger und E. Brunn, J. Org. Chem. 47, 661 (1982)

39) $M$. Schindler und $W$. Kutzelnigg, J. Am. Chem. Soc. 105, 1360 (1983).

40) $A$. Roedig und G. Märkl, Liebigs Ann. Chem. 636, 1 (1960).

41) T. J. Katz, R. J. Roth, N. Acton und E. J. Carnahan, Org. Synth. 53, 157 (1973)

42) Zur Synthese von 4 verwendeten wir bromidfreies Methyllithium von CHEMETALL, Frankfurt/Main.

43) G. L. Closs und K. D. Krantz, J. Org. Chem. 31, 638 (1966).

44) J. C. Stickler und W. H. Pirkle, J. Org. Chem. 31, 3444 (1966); R. C. Cookson, S. S. Gupte, I. D. R. Stevens und C. T. Watts, Org. Synth. 51, 121 (1971)

45) Benzobenzvalen ist ein nützlicher Trivialname. Der systematische Name des Benzvalens (Tricyclo[3.1.0.0 $\left.0^{2,6}\right]$ hex-3-en) eignet sich nicht zur Benzoanellierung, weshalb die Tetracyclokonstruktion benutzt wird.

46) F. M. Logullo, A. H. Seitz und L. Friedman, Org. Synth. 48, 12 (1968).

47) $I$. Murata und $K$. Nakasuji, Tetrahedron Lett. 1973, 47; R. M. Pagni und $C . R$. Watson, ebenda 1973, 59; C. R. Watson jr., R. M. Pagni, J. R. Dodd und J. E. Bloor, J. Am. Chem. Soc. 98, 2551 (1976).

48) J. Ipaktschi, Chem. Ber, 105, 1996 (1972).

49) D. H. Hunter und J. B. Stothers, Can. J. Chem. 51, 2884 (1973).

so) N. K. Wilson und J. B. Stothers, J. Magn. Reson. 15, 31 (1974). 